J. Math. Kyoto Univ.

10-3 (1970) $493-536$

\title{
On the modulus of continuity of sample functions of Gaussian processes
}

\author{
By \\ Norio Kôno \\ (Communicated by Prof. Yosida, April 24, 1970)
}

\section{Introduction}

It is interesting that many important properties of sample functions of $G . p .^{13}$ hold with probability 0 or 1 . For some class of G.p.'s the modulus of continuity of sample functions is one of such properties. Let $\{X(s) ; s \in D\}$ be a real valued G.p. with a parameter space $D$. We shall throughout this paper assume the following.

(A. 1) $D$ is a compact convex subset of $N$-dimensional Euclidean space containing an open set with the usual Euclidean metric $\|s-t\|^{2}=\sum_{i=1}^{N}\left(s_{i}-t_{i}\right)^{2}$.

(A. 2) $\{X(s) ; s \in D\}$ has the mean $E[X(s)]=0$ and with stationary increment $\sqrt{E\left[(X(s)-X(t))^{2}\right]}=\sigma(\|s-t\|)$, where $\sigma^{2}(x)$ $(\sigma(x) \equiv 0)$ is concave near the origin and $\sigma(x)$ is a non-decreasing continuous function that satisfies

$$
\int_{0}^{\infty} \sigma\left(e^{-x^{2}}\right) d x<+\infty .
$$

This condition guarantees that the G.p. has continuous sample functions by modification due to the theorem of X. Fernique [5]. So we shall assume that the G.p. has continuous sample functions.

Now we are led to introduce the concept of upper class and lower class with respect to the modulus of uniform continuity or local continuity of sample functions.

Let $\varphi(x)$ be a non-increasing continuous function satisfying

1) G.p. means Gaussian process. 
$\lim _{x, 0} \varphi(x)=+\infty$. Then, after P. Lévy [10], we shall say that $\varphi(x)$ belongs to the upper class $U^{\mu}(X)$ with respect to the modulus of unifrom continuity if there exists a positive constant $\delta(\omega)$, with probability 1 , such that $|X(s)-X(t)|<\sigma(\|s-t\|) \varphi(\| s-t||)$ holds for any $s, t$ with $\|s-t\|<\delta(\omega)$. We shall also say that $\varphi(x)$ belongs to the lower class $\mathcal{L}^{\prime \prime}(X)$ with respect to the modulus of uniform continuity if there exists no such positive constant with probability 1 . In the same manner we can define the upper class $\mathcal{V}^{\prime}(X)$ and the lower class $\mathcal{L}^{\prime}(X)$ with respect to the modulus of local continuity at any fixed point.

Investigations of the Hölder continuity of sample functions are formulated from our standpoint as follows: Sample functions of a G.p. are said to be uniform Hölder continuous of modulus $\varphi(x)$ if and only if $c \varphi(x) \in \mathcal{U}^{\prime \prime}(X)$ or $\in \mathcal{L}^{*}(X)$ according as $c>1$ or $0<c<1$ respectively. In a similar manner we can define the local Hölder continuity of modulus $\varphi(x)$. For some class of G.p.'s, however, we can only know properties that are weaker than the Hölder continuity in our sense, that is $c \varphi(x) \in \mathcal{V}^{\prime \prime}(X)$ or $\mathcal{L}^{\prime \prime}(X)$ $\left(U^{\prime}(X)\right.$ or $\left.\mathcal{L}^{\prime}(X)\right)$ provided $c>c_{1}$ or $c<c_{2}\left(c_{1}>c_{2}\right)$ respectively, but we emphasize that $c_{1}$ and $c_{2}$ must coincide from Kolmogorov's $0-1$ law as is explained in Section 6.

In case of Brownian motion, I. Petrovsky [14] proved the deciding condition that determines whether $\varphi(x)$ is of the class $U^{\prime}(B)$ or $\mathcal{L}^{\prime}(B)$ (Kolmogorov test). This alternative corresponds to the regular points for the boundary value problem of the heat equation. K. L. Chung-P. Erdös-T. Sirao [3] proved an analogous deciding condition for uniform continuity. T. Sirao [17] showed deciding conditions for the case of Brownian motion with multidimensional parameter.

In this paper we shall give each deciding condition which determines $\mathcal{U}^{\prime}(X)$ or $\mathcal{L}^{\mu}(X)$ and $\mathcal{V}^{\prime}(X)$ or $\mathcal{L}^{\prime}(X)$ for a class of $G$. $p$.'s characterized by the conditions on the increment variance $\sigma(x)$. That is, Theorems 3 and 7 state that if $\sigma(x)$ is a nearly regular varying function (n.r.v.f. Definition 2 in Section 2), then $\varphi(x)$ belongs to $\mathcal{U}^{\prime \prime}(X)$ or $\mathcal{L}^{\mu}(X)$ according as 


$$
I_{u}(\sigma, \phi)=\int_{+0}\left[\frac{x^{N \cdots 1} \exp \left\{-\frac{1}{2} \phi^{*}(x)\right\}}{\left.\left.\left[\sigma^{-1}\left(-\frac{\sigma(x)}{V}\right)\right]^{2 N} \frac{(x)}{\log / x}\right)\right]^{2}(x)} d x^{1)}\right.
$$

converges or diverges respectively. Also $\varphi(x)$ belongs to $V^{\prime}(X)$ or $\mathcal{L}^{\prime}(X)$ according as

$$
I_{I}(\sigma, \varphi)=\int_{+0}\left[\frac{x^{N-1} \exp \left\{-\frac{1}{2} \varphi^{2}(x)\right\}}{\left[\sigma^{-1}\left(\frac{\sigma(x)}{\sqrt{\log _{(2,} 1 / x}}\right)\right]^{N} \phi(x)} d x^{2}\right.
$$

converges or diverges respectively.

Recently, T. Sirao-H. Watanabe [18] have treated the G.p.'s with stationary increments on an interval for which $\sigma^{2}(x)$ satisfies the inequality

$$
c x^{\infty}(\log 1 / x)^{\beta} \leq \sigma^{2}(x) \leq c^{\prime} x^{\infty}(\log 1 / x)^{\beta}
$$

and is also concave near the origin. Their results have shown that the upper class and the lower class depend on $\alpha$ but not on $\beta$, that is, both classes depend only on the exponent of n.r.v.f. in this case. Here arises the question of whether or not the upper and lower classes are the same for any two G.p.'s with increment variances which are n.r.v.f. with the same exponent. Our theorems, however, state that both classes can be different even if corresponding $\sigma(x)$ 's are n.r.v.f. with the same exponent. For example, let $\left\{X_{i}(t) ; 0 \leq t \leq 1\right\}(i=1,2,3)$ be three G.p.'s with stationary increments which have increment variances $\sigma_{i}(x)$ such that

$$
\begin{aligned}
& \left.\sigma_{1}(x) \asymp x^{\infty}{ }^{3}\right), \\
& \sigma_{2}(x) \asymp x^{\alpha} \exp \left\{(\log 1 / x)\left(\log _{(\vartheta)} 1 / x\right)^{-\beta}\right\} \text {, } \\
& \sigma_{3}(x) \asymp x^{\infty} \exp \left\{(\log 1 / x)\left(\log _{(3)} 1 / x\right)^{-\gamma}\right\} \text {, } \\
& (0<\alpha \leq 1 / 2, \beta, \gamma>0) \text {. }
\end{aligned}
$$

1) By concavity and monotonicity of $\sigma^{2}(x), \sigma(x)$ has the inverse function near the origin. The integral $\int_{+0}$ means $\lim _{z \rightarrow 0} \int_{z}^{x}$ if $\sigma^{2}(x)$ is concave at $(0, u]$.

2) $\log _{(2)} x=\log \log x, \log (n) x=\log \log (n-1) x$.

3) $f(x)=g(x)$ means $0<\lim f(x) g(x) \leq \lim f(x) g(x)<\cdots$. 
Then we have

$$
\mathcal{V}^{\mu}\left(X_{1}\right)=\mathcal{V}^{\mu}\left(X_{2}\right) \text { and } \mathcal{L}^{\mu}\left(X_{1}\right)=\mathcal{L}^{\mu}\left(X_{2}\right)
$$

when $\beta \geq 1$, but these equalities never hold when $0<\beta<1$, and similarly we get

$$
\mathcal{U}^{\prime}\left(X_{1}\right)=\mathcal{U}^{\prime}\left(X_{3}\right) \text { and } \mathcal{L}^{\prime}\left(X_{1}\right)=\mathcal{L}^{\prime}\left(X_{3}\right)
$$

when $\gamma \geq 1$, but these equalities never hold when $0<\gamma<1$.

T. Kawada [8] proved an analogous deciding condition in case of G.p. with increment variance $E\left[(X(s)-X(t))^{2}\right]=\|s-t\|^{*}$ $(0<\alpha \leq 1)$ over $N$-dimensional Euclidean space. Many authers have investigated the Hölder continuity of sample functions [1], [13], [19].

We shall give in Theorems 1 and 2 the uniform Hölder continuity obtained by M. B. Marcus in case of a G.p. on an interval. It is remarkable, as is explained in Remarks 1 and 2, that if $\sigma(x)$ is a n.r.v.f. the modulus of uniform Hölder continuity equals $\sqrt{2 \log N_{x}(D)}$, where $N_{2}(D)$ is the minimal number of $\varepsilon-$ covering of $D$. This fact is valid even if $D$ is some infinite dimensional compact subset of a Hilbert space, (which contains the results of P.T. Strait [19]).

We shall also give some results on the modulus of local continuity of sample functions (Section 5).

Our theorems suggest to us that it is useful to investigate the modulus of continuity of sample functions of G.p.'s under some classification. Now we shall propose the following classification of G.p.'s. Let $\{X(s)\}$ be a G.p. satisfying our assumptions. Set

$$
\sigma(x)=F_{\sigma}(x) / \sigma(x) .
$$

where $F_{\sigma}(x)=\int_{0}^{\infty} \sigma\left(x e^{-u^{2}}\right) d u$.

Class I. $\{X(s)\}$ belongs to Class I if $\bar{\sigma}(x)$ is bounded.

Class II. $\{X(s)\}$ belongs to Class II if $\sigma(x)$ is not bounded but satisfies

$$
\lim _{x+0} \sigma(x) / \sqrt{\log 1 / x}=0 .
$$


Class III. $\{X(s)\}$ belongs to Class III if $\bar{\sigma}(x)$ satisfies

$$
\delta(x) \asymp \sqrt{\log 1 / x} .
$$

Class IV. The rest of the G.p.'s.

We shall briefly discuss in Section 3 the meaning of our classification and difference of classes. Also we shall give a classification if the function $\sigma(x)$ is a n.r.v.f. or a n.s.v.f. (Definition 4 in Section 2).

The content of each section is as follows.

1. Introduction.

2. Some notation and some definitions.

3. About our classification.

4. Theorems on the modulus of uniform continuity.

5. Theorems on the modulus of local continuity.

6. Preliminary lemmas.

7. Proofs of Theorems 1 and 2 .

8. Proofs of Theorems 3 and 4.

9. Proofs of Theorems 5 and 6.

10. Proofs of Theorems 7 and 8 .

11. Proofs of Propositions 1 4.

The autbor is greatly indebted to Professor T. Sirao, Professor H. Watanabe and Dr. I. Kubo for sending him their unpublished papers.

\section{Some notation and some definitions}

First we shall give the following modified definitions of regular varying function with exponent $\alpha>0$, nearly regular varying function, slowly varying function, and nearly slowly varying function due to J. Karamata [7].

Definition 1. Let $r(x)$ be a positive continuous function defined on a semi-closed interval $\left(0, u_{0}\right]$. Then $r(x)$ is called a regular varying function with exponent $\alpha(>0)(r . v . f .(\alpha))$ if it holds $\lim _{x \neq 0} r(t x) / r(x)=t^{a}$ for any $t>0$. 
Definition 2. We shall say that $f(x)$ is a nearly regular varying function with exponent $\alpha$ (n.r.v.f. $(\alpha)$ ) if there exists a r.v.f. $(\alpha) r(x)$ such that $r(x) \asymp f(x)$.

Definition 3. Let $s(x)$ be a function defined in Definition 1 . Then $s(x)$ is called a slowly varying function (s.v.f.) if it holds $\lim _{x \neq 0} s(t x) / s(x)=1$ for any $t>0$.

Definition 4. We shall say that $g(x)$ is a nearly slowly varying function (n.s.v.f.) if there exists a s.v.f. $s(x)$ such that $s(x) \asymp g(x)$.

It is well known (for instance [20]) that $r(x)$ is a r.v.f. $(\alpha)$ if and only if there exists a s.v.f. $s(x)$ such that $r(x)=x^{\alpha} s(x)$ and $s(x)$ is expressible as follows:

$$
s(x)=b(x) \exp \left\{-\int_{x}^{c} \frac{a(u)}{u} d u\right\} \quad\left(c \in\left(0, u_{0}\right)\right),
$$

where $a(x)$ and $b(x)$ are continuous functions such that $\lim _{x \rightarrow 0} a(x)=0$ and $\lim _{x \neq 0} b(x)>0$.

Definition 5. We shall say the function $a(x)$ of (2.1) a structure function of the s.v.f. or the n.s.v.f..

The following definition is useful to describe our assertions.

Definition 6. For two functions $f(x)$ and $g(x)$ defined near the origin, we shall denote by $f \gg g(x \downarrow 0)$ if there exists $u_{0}>0$ such that $f(x) \geq g(x)$ holds for any $0<x<u_{0}$. We shall say that $f_{\lambda} \gg g_{\lambda}(x \downarrow 0)$ holds uniformly if there exists a constant $u_{0}>0$ independent of $\lambda$ such that $f_{\lambda}(x) \geq g_{\lambda}(x)$ holds for any $0<x<u_{0}$. By an analogous way we shall define $a_{n} \gg b_{n}(n \rightarrow \infty)$ for two sequences $\left\{a_{n}\right\}_{n=1}^{\infty}$ and $\left\{b_{n}\right\}_{n=1}^{\infty}$.

Next we shall prepare some notation which are used repeatedly.

$$
\begin{aligned}
& \Phi(a)=\frac{1}{\sqrt{2}} \int_{a}^{\infty} e^{-u^{2} / 2} d u, \\
& \Phi_{r}(a, b)-\int_{a}^{\infty} \int_{b}^{\infty} \frac{1}{2 \pi \sqrt{1-\bar{r}^{2}}} \exp \left\{-\frac{x^{2}-2 r x y+y^{2}}{2\left(1-r^{2}\right)}\right\} d x d y,
\end{aligned}
$$




$$
\begin{aligned}
& F_{\sigma}(x)=\int_{0}^{\infty} \sigma\left(x e^{-u^{2}}\right) d u, \\
& G_{\sigma}(x)=\int_{x}^{\infty} \sigma\left(e^{-u^{2}}\right) d u, \\
& a \vee b=\max (a, b), \quad a \wedge b=\min (a, b) .
\end{aligned}
$$

Let $(S, \rho)$ be a compact metric space, and $K$ is a compact subset of $S$ with the diameter $d(K)$. Then we define

$$
\begin{gathered}
N_{\varepsilon}(K)=\inf \sharp\left\{K \subset \bigcup_{i=1}^{\dagger} A_{i} \quad \text { such that } d\left(A_{i}\right) \leq 2 \varepsilon\right\}, 1 \\
M_{\varepsilon}(K)=\sup \sharp\left\{\left(t_{1}, t_{2}, \cdots, t_{n}\right) \subset K \quad \text { such that } \rho\left(t_{i}, t_{j}\right)>\varepsilon\right. \\
\text { for any } i \neq j\} .
\end{gathered}
$$

We shall denote by $c_{i}(\varepsilon)$ a positive constant dependent on $\varepsilon$ and denote by $d_{i}$ an absolute constant.

\section{About our classification}

First we shall give a classification if the function $\sigma(x)$ is a n.r.v.f. or a n.s.v.f..

Proposition 1. If $\sigma(x)$ is a n.r.v.f., then the process is of Class I, and if $\sigma(x)$ is a n.s.v.f., it is not of Class $I$.

Proposition 2. Assume that $\sigma(x)$ is a n.s.v.f. with a structure function $a(x)$ which satisfies the following two conditions:

$$
a(x) \gg \frac{\delta}{\log 1 / x} \quad(x \downarrow 0)
$$

holds for any $\delta>0$.

(ii) There exists a constant $c_{3}>0$ such that

$$
a\left(x^{2 \cdot t}\right) \gg c_{3} a(x) \quad(x \downarrow 0)
$$

uniformly for any $0 \leq \varepsilon \leq 1$. Then the process is of Class II.

Proposition 3. If $\sigma(x)$ is a n.s.v.f. with a structure function $a(x)$ such that

1) $=I$ denotes the cardinal number of $I$. 


$$
\frac{c_{4}}{\log 1 / x} \gg a(x) \gg \frac{c_{5}}{\log 1 / x} \quad(x \downarrow 0),\left(c_{4}>c_{5}>1 / 2\right),
$$

then the process is of Class III.

Proposition 4. Assume that $\sigma(x)$ is a n.s.v.f. with a structure function $a(x)$ such that

$$
\begin{aligned}
& \frac{1}{2 \log 1 / x}+\frac{1}{(\log 1 / x)\left(\log _{(2)} 1 / x\right)}+\cdots+\frac{1}{(\log 1 / x) \cdots\left(\log _{(m-1)} 1 / x\right)} \\
& +\frac{c_{6}}{(\log 1 / x) \cdots\left(\log _{(m)} 1 / x\right)} \gg a(x) \gg \frac{1}{2 \log 1 / x}+\frac{1}{(\log 1 / x)\left(\log _{(2)} 1 / x\right)} \\
& +\cdots+\frac{1}{(\log 1 / x) \cdots\left(\log _{(m-1)} 1 / x\right)}+\frac{c_{7}}{(\log 1 / x) \cdots\left(\log _{(m)} 1 / x\right)}, \\
& (x \downarrow 0),\left(c_{6}>c_{7}>1\right) .
\end{aligned}
$$

\begin{tabular}{|c|c|}
\hline Class & $\sigma(x) \asymp$ \\
\hline I & $\begin{array}{l}\text { n.r.v.f., } x^{\alpha}(\log 1 / x)^{\beta} \quad(0<\alpha,-\infty<\beta<+\infty) \\
x^{\alpha} \exp \left\{ \pm(\log 1 / x)^{\beta}\right\} \quad(0<\alpha,-\infty<\beta<1)\end{array}$ \\
\hline II & $\exp \left\{-(\log 1 / x)^{\beta}\left(\log _{(2)} 1 / x\right)^{y}\right\}$ \\
\hline III & $(\log 1 / x)^{-\gamma}\left(\log _{(2)} 1 / x\right)^{\delta} \quad(\gamma>1 / 2,-\infty<\delta<+\infty)$ \\
\hline IV & 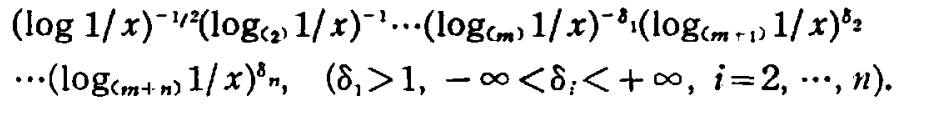 \\
\hline
\end{tabular}

Then the process is of Class IV.

These propositions are proved in Section 11. Here we shall give some examples.

Now we shall briefly discuss the meaning of our classification and the difference of classes.

In case of G.p.'s with stationary increments on an interval, it follows from Theorem 1 and Theorem 2 that if a G.p. is of Class I or II the sample functions are uniform Hölder continuous of modulus $\sqrt{2} \overline{\log 1 / x}$, while if the process is of Class III the sample functions are uniformly Hölder continuous of modulus $\sqrt{c \log 1 / x}$ which is of $\mathcal{V}^{\mu}(X)$ or $\mathcal{L}^{*}(X)$ according as $c>c_{\mathrm{g}} \geq 2$ or 
$c<2$ respectively. If, in particular, we assume that $\sigma(x)$ is a n.r.v.f. the process belongs to Class $\mathrm{I}$ and we have the conditions that decide whether $\varphi(x)$ is of $\mathcal{U}^{\prime \prime}(X)$ or $\mathcal{L}^{\prime \prime}(X)$ and $\mathcal{U}^{\prime}(X)$ or $\mathcal{L}^{\prime}(X)$ as decribed already.

The crucial difference between Class I and Class II may be seen by a particular choice of $\phi(x)$ as follows. If $\sigma(x)$ is a n.r.v.f. (i.e. the G.p. is of Class I) we see that $\varphi(x)=\sqrt{2 \log 1 / x} \bar{x} \overline{\log } \overline{\alpha_{2}} 1 / x$ belongs to $\mathcal{U}^{\mu}(X)$ or $\mathcal{L}^{\mu}(X)$ depending on the constant $c$. While if $\sigma(x)$ is a n.s.v.f. with a structure function $a(x)$ the process is of Class II only under certain conditions on $a(x)$ and $\varphi(x)=$ $\sqrt{2 \log 1 ; x+c a(x)^{-1}} \log _{c_{2}} 1 / x \in \mathcal{U}^{u}(X)$ or $\in \mathcal{L}^{\mu}(X)$ (Theorem 4). Deciding conditions for $\mathcal{U}^{\mu}(X)$ or $\mathcal{L}^{u}(X)\left(\mathcal{U}^{\prime}(X)\right.$ or $\left.\mathcal{L}^{\prime}(X)\right)$ of Class II IV are unknown.

\section{Theorems on the modulus of uiform continuity}

First we shall give the uniform upper bound of sample functions.

Theorem 1. Let $\{X(s) ; s \in D\}$ be a G.p. satisfying our assumptions (A.1), (A.2) and assume that $\sigma(x) \sqrt{\log 1} / \bar{x}$ is a non-decreasing function near the origin. Then

$$
\varlimsup_{\| s i t: i \neq 0} \frac{|X(s)-X(t)|}{\sigma(\| s-t \mid) \varphi_{z}(\|s-t\|)} \leq 1
$$

holds with probability one for any $\varepsilon>0$, where

$$
p_{q}(x)=\sqrt{(\overline{2}+\varepsilon) N \log 1 / x}+c_{9}(\varepsilon) G_{\sigma}(\sqrt{N \overline{\log } 1 / x)} / \sigma(x) .
$$

Remark 1. Theorem 1 is still valid under the non-decreasingness of $\sigma(x) \vee \bar{H}(\overline{\log 1 / x)} \text { and the following conditions (A. } 1)^{(*)}$, $(\text { A. } 2)^{(*)}$ instead of (A. 1), (A. 2) by substituting

$$
\left.\varphi_{\mathrm{e}}(x)=\sqrt{(4+\varepsilon) H\left(\overline{\log } \overline{1 / x}+c_{10}(\varepsilon) G_{\sigma}^{H}(\sqrt{H(\log } \overline{1 / x})\right.}\right) / \sigma(x)
$$

where $G_{\sigma}^{H}(x)=\int_{x}^{\infty} \sigma\left(\exp \left\{-H^{-1}\left(u^{2}\right)\right\}\right) d u$.

$(A .1)^{(*)}$ The parameter space $(D, \rho)$ is a compact centered metric

1) For any two points $s_{1}, s_{2} \in D$ there exists $s_{0} \in D$ such that $\rho\left(s_{0}, s_{1}\right)=\rho\left(s_{0}, s_{1}\right)$ $=1 / 2 \rho\left(s_{1}, s_{2}\right)$. 
space $^{1)}$ satisfying

$\exp \{(1+\delta) H(\log 1 / \varepsilon)\} \gg N_{\varepsilon}(D) \gg \exp \{(1-\delta) H(\log 1 / \varepsilon)\} \quad(\varepsilon \downarrow 0)$ for any $\delta>0$, where $1 / H(1 / x)$ is a strictly increasing r.v.f. $(\mu)$ $(\mu \geq 1)$.

$(\text { A. } 2)^{(*)}$

$$
G_{\sigma}^{H}(0)<+\infty .
$$

Furthermore assume that $(D, \rho)$ satisfies

$(\text { A. } 1)^{(* *)} \quad \lim _{\varepsilon \neq 0} \frac{N_{\varepsilon}(B(t, a \varepsilon))}{N_{\mathrm{g}}(D)}=0, \quad(B(t, r)=\{s \in D ;\|t-s\| \leq r\})$,

uniformly in $t \in D$ for any $a>1$. Then we can choose $\varphi_{\mathrm{g}}(x)$ instead of (4.3) as follows:

(4.4) $\varphi_{\mathrm{e}}(x)=\sqrt{(2+\varepsilon) H(\log 1 / x)}+c_{11}(\varepsilon) G_{\sigma}^{H}(\sqrt{H(\log 1 / x)}) / \sigma(x)$.

Corollary 1. When a G.p. $\{X(s) ; s \in D\}$ belongs to Class $I, I I$ or III and satisfies the assumption of Theorem 1, we have

$$
\varlimsup_{1: s-\lrcorner\|+\| \overline{\sigma(\| s-t||)}}-\frac{|X(s)-X(t)|}{2 N \log 1 /\|s-t\|} \leq c_{12}
$$

with probability one. Furthermore if the process belongs to Class I or II we can choose $c_{12}=1$, but if the process belongs to Class III it is an open problem whether $c_{12}=1$ or not.

Corollary 2. If a G.p. $\{X(s): s \in D\}$ satisfies the conditions of Proposition 4, we have

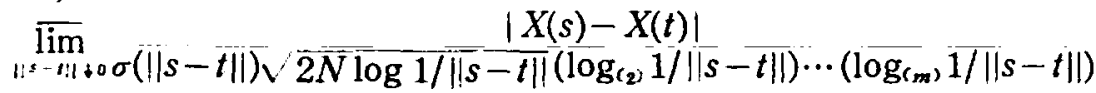

$$
\begin{aligned}
& \leq c_{13}<+\infty
\end{aligned}
$$

with probability one.

Next we shall give the uniform lower bound of sample functions.

Theorem 2. Let $\{X(s) ; s \in D\}$ be a G.p. satisfying our assumptions (A.1) and (A.2). Then we have

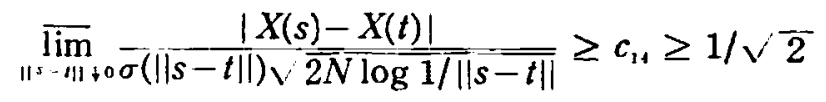


with probability one. Furthermore if we assume that $\sigma(x)$ is a n.r.v.f. we can set $c_{14}=1$.

Remark 2. Theorem 2 can be extended as follows. If the parameter space $(D, \rho)$ satisfies $(A .1)^{(*)}$ and have the four point property ${ }^{13}$ we have

$$
\varlimsup_{\mid i^{s}-f_{i \mid+0} \sigma(\mid)} \frac{|X(s)-X(t)|}{|s-t| \mid) \sqrt{2 H(\log 1 / \| s-t||)}} \geq c_{1 s} \geq 1 / \sqrt{2}
$$

with probability one. Furthermore if we assume that $\sigma(x)$ is a n.r.v.f. we can set $c_{15}=1$.

For example define $D=\left\{\left(a_{1}, a_{2}, \cdots\right) \in l: \sum_{k=n}^{\infty} a_{k}^{2} \leq 2^{-n}\right.$ for any $\left.n\right\}$ with usual $l^{2}$-metric. Then $N_{\mathrm{s}}(D)$ satisfies $(\text { A. } 1)^{(*)}$ for $H(x)=$ $x^{2} / 2([11])$.

Remark 3. If $D=[0,1]$, we can set $c_{14}=1$ ([13]).

Now we shall give a deciding condition for uniform upper class or lower class.

Theorem 3. Let $\{X(s) ; s \in D\}$ be a G.p. satisfying our assumptions (A.1) and (A.2). Furthermore we assume that $\sigma(x)$ is a n.r.v.f.. Then we have

$$
\varphi \in \mathcal{Q}^{u}(X) \quad \text { if } \quad I_{u}(\sigma, \varphi)<+\infty
$$

and

$$
\varphi \in \mathcal{L}^{\mu}(X) \quad \text { if } \quad I_{u}(\sigma, \varphi)=+\infty \text {. }
$$

As the corollary of Theorem 3 , we shall give a condition of invariance of $\mathcal{U}^{\mu}(X)$ and $\mathcal{L}^{\prime \mu}(X)$ which we have pointed out in Introduction.

Let $\left\{X_{i}(s) ; s \in D\right\}(i=1,2)$ be two G.p.'s satisfying our assumptions (A. 1) and (A. 2). Set $\sigma_{1}(x) \asymp x^{*}(0<\alpha \leq 1 / 2)$ and $\sigma^{2}(x) \asymp$ $x^{\infty} \tau(x)$, where $\tau(x)$ is a s.v.f. with a structure function $a(x)$. Then there exists a s.v.f. $\hat{\tau}(x)$ such that

$$
\sigma_{2}^{-1}\left(\frac{\sigma_{2}(x)}{\sqrt{\log 1 / x}}\right) \asymp x(\log 1 / x)^{-1 / 2 \infty} \hat{\tau}(x)
$$

1) Any four points of $D$ can be imbeded in 3-dimensional Euclidean space without changing their metrics each other. 
Corollary 3. If

$$
\hat{f}(x) \asymp c_{16}
$$

then we have

$$
\mathcal{U}^{\mu}\left(X_{1}\right)=\mathcal{U}^{\mu}\left(X_{2}\right) \text { and } \mathcal{L}^{*}\left(X_{1}\right)=\mathcal{L}^{\mu}\left(X_{2}\right) .
$$

In particular,

$$
\left|a(x) \log _{(2)} 1 / x\right| \leq c_{17}
$$

guarantees the relation (4.11).

Corollary 4. On the other hand, (4.12) does not hold if $a(x)$ satisfies the condition (ii) of Proposition 2 and

$$
\left|a(x) \log _{(2)} 1 / x\right| \gg c_{1 \mathrm{~B}} \log _{(3)} 1 / x \quad(x \downarrow 0) .
$$

In case of $\sigma(x)$ being not a n.r.v.f. we have only restricted results as follows.

Theorem 4. If $\sigma(x)$ is a n.s.v.f. with a structure function $a(x)$ which satisfies the following conditions:

(i) $a(x)$ is non-decreasing and there exists a constant $c_{10}(\delta)$ satisfying $\lim _{\delta, 0} c_{19}(\delta)=1$ such that

$$
a\left(x^{1+8}\right) \gg c_{19}(\delta) a(x) \quad(x \downarrow 0)
$$

holds uniformly.

(ii) There exists a constant $1>\beta>0$ such that

$$
a(x) \gg(\log 1 / x)^{\beta-1} \quad(x \downarrow 0) .
$$

Then

$$
\begin{aligned}
\varphi_{\mathrm{g}}(x)= & \sqrt{2 N \log 1 / x+2 N(1+\varepsilon)\left(\log _{\left(_{2}\right)} 1 / x\right) / a(x)} \\
& \in \mathcal{V}^{\mu}(X) \quad \text { if } \varepsilon>0,
\end{aligned}
$$

and

$$
\in \mathcal{L}^{u}(X) \text { if } \varepsilon<0 .
$$

The conditions (i) and (ii) imply that this process is of Class II by Proposition 2 . 


\section{Theorems on the modulus of local continuity}

In this section we shall give some theorems corresponding to the case of uniform continuity. Theorem 5 and 6 are concerned with the local Hölder continuity. M. B. Marcus [13] has proved about this in case of $D=[0,1]$. We shall adjust his results in case of $\sigma(x)$ satisfying some regularity conditions. Theorem 7 gives a deciding condition that determines whether $\varphi(x)$ belongs to $\mathcal{U}^{\prime}(X)$ or $\mathcal{L}^{\prime}(X)$.

Let $\{X(s) ; s \in D\}$ be a G.p. with our assumptions (A. 1) and (A. 2).

Theorem 5. (i) If $\sigma(x)$ is a n.r.v.f., then for any fixed point $s_{0} \in D$.

$$
\overline{\lim }_{\left\|=s_{0}\right\|+0} \frac{\left|X(s)-X\left(s_{0}\right)\right|}{\sigma\left(\| s_{0}-s||\right) \sqrt{2 \log } 1 /|| \overline{s_{0}-s||}} \leq 1
$$

holds with probability one.

(ii) Assume that $\sigma(x)$ is a n.s.v.f. with a structure function $a(x)$ which satisfies following condition:

a) $a(x)$ is non-decreasing and satisfies

$$
a\left(x^{1+8}\right) \gg c_{20} a(x) \text { uniformly for } 0 \leq \varepsilon \leq 1 \text {. }
$$

b)

$$
a(x) \gg \frac{\gamma}{\sqrt{\log 1 / x}}, \quad(x ! 0)(\gamma>1 / 2) \text {. }
$$

Then

$$
\varlimsup_{\| s \rightarrow s_{0} !+\circ 0} \frac{\left|X(s)-X\left(s_{0}\right)\right|}{\sigma\left(\left\|s-s_{0}\right\|\right) \varphi\left(\left\|s-s_{0}\right\|\right)} \leq c_{21}
$$

holds with probability one, where

$$
\varphi(x)=\sqrt{\log _{(2)} 1 / x \vee 1 / a(x)},
$$

(iii) If $\sigma(x)$ is n.s.v.f. with a non-decreasing structure function $a(x)$ satisfying the condition of Proposition 4, then

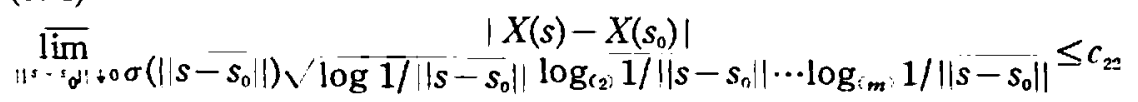

holds with probability one. 
Next we shall give the local lower bound of sample functions of a G.p..

Theorem 6. (i) If $\sigma(x)$ is a n.r.v.f.,

$$
\varlimsup_{\left\|s-s_{0}\right\|+0} \frac{\left|X(s)-X\left(s_{0}\right)\right|}{\sigma\left(\| s-s_{0} \mid\right) \sqrt{2 \log _{(2)}} \overline{1 /\left\|s-s_{0}\right\|}} \geq 1
$$

holds with probability one.

(ii) Assume that $\sigma(x)$ is a n.s.v.f. of a structure function $a(x)$ satisfying the following condition:

$$
0 \leq a(x+h)-a(x) \ll c_{34} h a(x) / x \quad(x \downarrow 0),
$$

holds uniformly for $h>0$. Then

$$
\lim _{\left\|s-s_{0}\right\|+00} \frac{\left|X(s)-X\left(s_{0}\right)\right|}{\sigma\left(\left\|s-s_{0}\right\|\right) \varphi\left(\left\|s-s_{0}\right\|\right)} \geq c_{2 s}>0
$$

holds with probability one, where $\varphi(x)$ is given by (5.3).

Now we shall give a deciding condition which determines $q^{\prime \prime}(X)$ or $\mathcal{L}^{\prime}(X)$ for the same class of G.p.'s as Theorem 3. This theorem includes the cases of Theorem 5, (i) and Theorem 6, (i).

Theorem 7. Under the same conditions of Theorem 3, we have and

$$
\begin{array}{lll}
\phi \in \mathcal{U}^{\prime}(X) & \text { if } \quad I_{l}(\sigma, \phi)<+\infty, \\
\varphi \in \mathcal{L}^{\prime}(X) & \text { if } \quad I_{1}(\sigma, \phi)=+\infty .
\end{array}
$$

We have the following corollaries of Theorem 7 by the same way as Corollaries 3 and 4.

Corollary 5. Under the same situation of Corollary 3, we have

$$
\sigma_{2}^{-1}\left(\frac{\sigma_{2}(x)}{\log _{(2)} 1 / x}\right) \asymp x\left(\log _{(2)} 1 / x\right)^{-1 / 2 \infty} \widetilde{\tau}(x),
$$

where $\tilde{\tau}(x)$ is a s.v.f. and if

$$
\tilde{\tau}(x) \asymp c_{36}
$$

we get

$$
\mathcal{U}^{\prime}\left(X_{1}\right)=\mathcal{U}^{\prime}\left(X_{2}\right) \text { and } \mathcal{L}^{\prime}\left(X_{1}\right)=\mathcal{L}^{\prime}\left(X_{2}\right)
$$


On the modulus of continuity of sample functions 507

In particular,

$$
\left|a(x) \log _{(3)} 1 / x\right| \leq c_{27}
$$

guarantees the relation (5.12).

Corollary 6. On the other hand, (5.12) does not hold if $a(x)$ satisfies the condition (ii) of Proposition 2 and

$$
\left|a(x) \log _{\left(_{3}\right.}, 1 / x\right| \gg c_{3 \mathrm{~B}} \log _{\left(_{8}\right)} 1 / x \quad(x \downarrow 0) .
$$

In case $\sigma(x)$ being not a n.r.v.f. we have only restricted results as follows.

Theorem 8. Assume that $\sigma(x)$ is a n.s.v.f. with a structure function $a(x)$ which satisfies the condition (i) of Theorem 4 and $a$ following condition:

(i) There exists a positive constant $1>\delta>0$ such that

$$
a(x) \gg\left(\log _{(2)} 1 / x\right)^{s-1} \quad(x \downarrow 0) .
$$

Then

$$
\begin{aligned}
\varphi_{\mathrm{s}}(x)= & \sqrt{2 \log _{\mathfrak{C}_{2}} 1 / \overline{x+N(1+\varepsilon)\left(\log _{(3} 1 / x\right) / a(x)}} \\
& \in \mathcal{V}^{\prime}(X) \quad \text { if } \varepsilon>0
\end{aligned}
$$

and

$$
\in \mathcal{L}^{\prime}(X) \quad \text { if } \varepsilon<0 \text {. }
$$

\section{Preliminary lemmas}

Before we prove the theorems, we shall prepare some useful lemmas.

Lemma 1 (0-1 law). Let $\varphi(x)$ be a continuous function satisfying $\lim _{x \downarrow+\infty} \varphi(x)=+\infty$. Then the random variables

$$
\varlimsup_{\|s-t\| 1 * \sigma} \frac{|X(s)-X(t)|}{(|| s-t \mid) \varphi(|| s-t \mid i)}
$$

and

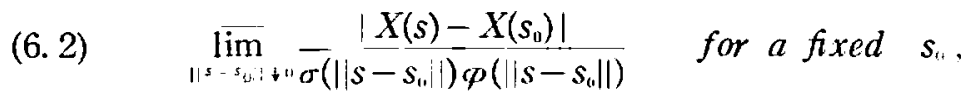

are constants with probability one. 
The proof of Lemma 1 is based on the fact that $\{X(s)\}$ is expanded uniformly in $s$ with probability one in the form $\sum_{n=1}^{\infty} \sqrt{\lambda_{n}} \varphi_{n}(s) X_{n}(\omega)$, where $\left\{\lambda_{n}\right\},\left\{\varphi_{n}(s)\right\}$ are eigen values and normalized eigen functions respectively of following integral equation,

$$
\lambda \varphi(s)=\int_{D} E[X(s) X(t)] \varphi(t) d t . \quad \text { (see [6], [21]). }
$$

By the definition of upper class or lower class and $I_{u}(\sigma, \varphi)$ or $I_{l}(\sigma, \varphi)$ we have

Lemma 2. (i) If $\varphi_{1} \in \mathcal{U}^{*}(X)\left(\mathcal{U}^{\prime}(X)\right)$ and $\varphi_{2} \gg \varphi_{1}$ then $\varphi_{2} \in$ $\mathcal{V}^{\mu}(X)\left(q^{\prime \prime}(X)\right)$.

(ii) $\phi_{3} \in \mathcal{L}^{\prime \prime}(X)\left(\mathcal{L}^{\prime}(X)\right)$ and $\phi_{3} \gg \varphi_{4}$, then $\varphi_{1} \in \mathcal{L}^{\prime \prime \prime}(X)\left(\mathcal{L}^{\prime}(X)\right)$.

(iii) If $I_{u}(\sigma, \varphi)<+\infty$, we have $\phi \gg \sqrt{N \log 1 / x}$ and $I_{u}\left(\sigma, \varphi_{s}\right)<+\infty$ by setting $\varphi_{5}=(\varphi \vee \vee \bar{N} \overline{l o g} 1 / x) \wedge \vee \overline{3} \overline{N \log 1 / x}$.

(iv) If $I_{u}(\sigma, \varphi)=+\infty$, then $I_{u}\left(\sigma, \varphi_{s}\right)=+\infty$ and if $\varphi_{5} \in \mathcal{L}^{u}(X)$ then $\varphi \in \mathcal{L}^{\mu}(X)$.

(v) The similar facts to (iii) and (iv) hold for $I_{l}(\sigma, \varphi)$.

The proof of Lemma 2 is in the same way as that of $T$. Sirao [17].

Lemma 3 (Borel-Cantelli). Let $\left\{A_{n}\right\}$ be a sequence of events.

(i) If $\sum_{n=1}^{\infty} P\left(A_{n}\right)<+\infty$, then $P\left(\varlimsup_{n \rightarrow \infty} A_{n}\right)=0$.

(ii) a) $\sum_{n=1}^{\infty} P\left(A_{n}\right)=+\infty$,

b) For each $n$ there exists finite subsequence $I_{n}=\left\{n \leq n_{1}<n_{2}<\cdots\right.$ $\left.<n_{i(n)}\right\}$ such that

(b. 1) $\sum_{i \in I_{n}} P\left(A_{n} \cap A_{i}\right) \leq d_{1} P\left(A_{n}\right)$

(b. 2) $P\left(A_{n} \cap A_{i}\right) \leq d_{2} P\left(A_{n}\right) P\left(A_{i}\right)$ if $i \notin I_{n}, i \geq n$,

then $P\left(\varlimsup_{n \rightarrow \infty} A_{n}\right) \geq \frac{1}{d_{2}}$.

The proof is obvious from Shwarz' inequality (c.f. [13] Lemma 1).

Lemma 4. (i) $[12$, p. 4$]$ 


$$
\frac{e^{-x^{2} / 2}}{\sqrt{2 \pi}(x+1 / x)} \leq \Phi(x) \leq \frac{e^{-x^{2} / 2}}{\sqrt{2 \pi} x} \quad(x>0)
$$

(ii) $[3$, Lemma 3]

$$
\Phi_{r}(a, b) \leq d_{3} \Phi(a) \Phi(b) \quad(a, b>0)
$$

for any $-1<r<1 / a b$, where $d_{3}$ is an absolute constant independent of $a, b$ and $r$.

(iii) $[3$, Lemma 4]

$$
\Phi_{r}(a, b) \leq d_{1} \exp \left\{-\frac{1-r}{4} b^{2}\right\} \Phi(a)
$$

for any $b \geq a \geq 0$ and $1>r \geq 0$.

(iv) $[17$, Lemma 2]

$$
\int_{-\infty}^{a} \int_{0}^{\infty} \frac{1}{\sqrt{1-r^{2}}} \exp \left\{-\frac{x^{2}-2 r x y+y^{2}}{2\left(1-r^{2}\right)}\right\} d x d y
$$

is a non-increasing function of $r$ for fixed $a$ and $b(0<a<b)$.

(v) $[2$, p. 508] (Slepian)

Let $R=\left\{r_{i, j}\right\}$ be a $N \times N$ symmetric positive definite matrix with $r_{i i}=1(i=1,2, \cdots, N)$. Define

$$
Q(c, R)=\int_{-\infty}^{c} \cdots \int_{-\infty}^{c} \frac{1}{\sqrt{\operatorname{det} R}} \exp \left\{-\frac{1}{2}\left(x, R^{-1} x\right)\right\} d x_{1} \cdots d x_{N} .
$$

Then $Q(c, R)$ is an increasing function of the arguments $r_{i, j}$.

Lemma 5. Let $s(x)$ be a s.v.f.. Then it follows by (2. 1) that for any $\varepsilon>0$ there exists a constant $c(\varepsilon)>0$ such that

$$
c(\varepsilon) t^{-*} \leq \frac{s(t x)}{s(x)} \leq c(\varepsilon) t^{\mathrm{z}}
$$

for any $t \geq 1,0<x t \leq d_{5}$.

Lemma 6. Let $f(x)$ be a concave function which is nondecreasing with $f(0)=0$.

$$
\begin{aligned}
& \left|f\left(x_{1}\right)+f\left(x_{2}\right)-f\left(x_{3}\right)-f\left(x_{1}\right)\right| \leq f\left(\left|x_{1}+x_{2}-x_{3}-x_{1}\right|\right) \\
& f\left(x_{1}\right)+f\left(x_{2}\right)-f\left(x_{3}\right) \geq f\left(x_{1}+x_{2}-x_{3}\right)
\end{aligned}
$$

if $x_{1} \div x_{2} \geq x_{3} \geq x_{1} \vee x_{2}$. 
Lemma 7. For any 4 points $A, B, A^{\prime}$ and $B^{\prime}$ of Euclitean space,

$$
\left|\overline{A B^{\prime}}+\overline{A^{\prime} B}-\overline{A A^{\prime}}-\overline{B B^{\prime}}\right| \leq 4 \overline{A B} \cdot \overline{A^{\prime} B^{\prime}} / R,
$$

where $R=\max \left\{\overline{A A^{\prime}}+\overline{A^{\prime} B}, \overline{A B^{\prime}}+\overline{B^{\prime} B}, \overline{A^{\prime} A}+\overline{A B^{\prime}}, \overline{B^{\prime} B}+\overline{A^{\prime} B}\right\}$

(ii) If $\overline{A B^{\prime}} \geq \overline{A A^{\prime}}$ and $\overline{A^{\prime} B} \geq \overline{B B^{\prime}}$ then

$$
\overline{A B^{\prime}}+\overline{A^{\prime} B}-\bar{A} \overline{A^{\prime}}-\bar{B} B^{\prime} \leq 2 \overline{A B} \cdot \overline{A^{\prime} B^{\prime}} / r
$$

where $r=\min \left\{\overline{A A^{\prime}}+\bar{A} \overline{B^{\prime}}, \overline{A^{\prime} B}+\bar{B} \overline{B^{\prime}}\right\}$.

$\overline{P Q}$ denotes the distance of $P$ and $Q$.

Lemma 8 [9]. Let $D \subset R^{N}$ be a compact convex subset which contains an open subset. Then for any $B(t, r)=\{s \in D:\|s-t\| \leq r\}$, $t \in D$,

$$
d_{6}(r / \varepsilon)^{N} \geq N_{6}(B(t, r)) \geq d_{7}(r / \varepsilon)^{N},
$$

where $d_{6}$ and $d_{7}$ are constants independent of $r$ and $\varepsilon$.

Lemma $9[9] . \quad M_{2 \xi}(\cdot) \leq N_{\xi}(\cdot) \leq M_{\xi}(\cdot)$.

\section{Proofs of Theorems 1 and 2}

First we shall prove Theorem 1 . We can choose $p>1$ such that $2 p^{2}-1<1+\varepsilon / 2$. Set

$$
\begin{aligned}
\delta_{n}= & \exp \left\{-p^{n}\right\}, \varepsilon_{n}=\delta_{n+2} / 6, \varphi(x)=\sqrt{2\left(2 p^{2}-1\right) N \log 1 / x}, \\
I_{n}= & \left\{(i, j, k) ; k=n-1, n,\left\|t_{i}^{(n)}-t_{j}^{(k)}\right\| \leq \delta_{n}\right\} \quad \text { and } \\
E_{i, j}^{n, k}= & \left\{\omega ;\left|X\left(t_{i}^{(n)}\right)-X\left(t_{j}^{(k)}\right)\right| \geq \sigma\left(\left\|t_{i}^{(n)}-t_{j}^{(k)}\right\|\right)\right. \\
& \left.\times \varphi\left(\left\|t_{i}^{(n)}-t_{j}^{(k)}\right\|\right)\right\}, \quad\left((i, j, k) \in I_{n}\right),
\end{aligned}
$$

where $\left\{t_{i}^{(n)}, i=1,2, \cdots, N_{t_{n}}(D)\right\}$ is a minimal $\varepsilon_{n}$-net of $D$. Then it follows from Lemma 4 , (i) and Lemma 8 that

$$
P\left(E_{, j}^{\prime, j}\right) \leq c_{3 n} \frac{\delta_{n}^{\left(2 p^{2}-1\right) N}}{\sqrt{\log } \overline{1 / \overline{\delta_{n}}}}
$$

and

$$
\oiint I_{n} \leq c_{31} \delta_{n}^{N} \varepsilon_{n}^{-2 N} \leq c_{32} \delta_{n}^{1-2 p^{2} 3 N} .
$$

Therefore combining (7.2) and (7.3) we have 


$$
\sum_{n}^{\infty} \sum_{(i, j, n) \in I_{n}} P\left(E_{i, j}^{n, k}\right) \leq \sum_{n}^{\infty} c_{30} \cdot c_{32} / v \overline{\log } 1 / \overline{\delta_{n}}<+\infty .
$$

Using Borel-Cantelli Lemma, there exists a integer $n_{0}(\omega)$, with probability one, such that

$$
\left|X\left(t_{i}^{(n)}\right)-X\left(t_{j}^{(k)}\right)\right| \leq \sigma\left(\left\|t_{i}^{(n)}-t_{j}^{(k)}\right\|\right) \varphi\left(\| t_{i}^{(n)}-t_{j}^{(n)} \mid i\right)
$$

holds for any $(i, j, k) \in I_{n}, n \geq n_{0}$.

Denote $\left\{t_{i(n)}^{(n)}\right\}_{n=n_{0}}^{\infty}$ be a sequence such that $\left\|s-t_{(n)}^{(n)}\right\| \leq 3 \varepsilon_{n}$ for a fixed $s \in D$. From the inequality $\left\|t_{i(n+1)}^{(n+1)}-t_{((n))}^{(n)}\right\| \leq\left\|t_{(n+1)}^{(n+1)}-s\right\|$ $+\left\|s-t_{l(n)}^{(n)}\right\| \leq 3 \varepsilon_{n+1}+3 \varepsilon_{n} \leq 6 \varepsilon_{n}=\delta_{n+2}<\delta_{n+1}$, we have $(i(n+1), i(n), n)$ $\in I_{n-1}$. Hence it follows from (7.5) and monotonicity of $\sigma(x) \sqrt{\log 1 / x}$ that

$$
\begin{gathered}
\left|X(s)-X\left(t_{i(k)}^{(n)}\right)\right| \leq \sum_{k=1}^{\infty}\left|X\left(t_{i(k+1)}^{(k+1)}\right)-X\left(t_{i(k)}^{(k)}\right)\right| \\
\leq \sum_{k=1}^{\infty} \sigma\left(\delta_{k+2}\right) \varphi\left(\delta_{k+2}\right) .
\end{gathered}
$$

But there exists a $c_{33}(p)$ such that

$$
\varphi\left(\delta_{k+2}\right) \leq c_{33}(p)\left(\sqrt{\log 1 / \delta_{k-2}}-\sqrt{\log } \overline{1 / \delta_{k+1}}\right),
$$

therefore we have

$$
\sum_{k \rightarrow 7}^{\infty} \sigma\left(\delta_{k+2}\right) \varphi\left(\delta_{k+2}\right) \leq c_{33}(p) G_{\sigma}\left(\sqrt{\log 1 / \delta_{n+1}}\right) .
$$

While for any $s, t \in D$ satisfying $\delta_{n+1} \leq\|s-t\| \leq \delta_{n}\left(n \geq n_{0}\right)$, there exists $t_{1_{1}}^{(n)}, t_{1_{2}}^{(n)}, t_{0}$ such that

$$
\begin{aligned}
& \left\|s-t_{i_{1}}^{(n)}\right\| \leq \varepsilon_{n}, \quad\left\|t_{i_{1}}^{(n)}-t_{0}\right\|=\|s-t\|-\varepsilon_{n}, \\
& \left\|t_{0}-t_{i_{2}}^{(n)}\right\| \leq \varepsilon_{n} .
\end{aligned}
$$

Thus we have

$$
\begin{aligned}
&|X(s)-X(t)| \leq\left|X(s)-X\left(t_{i_{1}}^{(n)}\right)\right|+\left|X\left(t_{i_{1}}^{(n)}\right)-X\left(t_{i_{2}}^{(n)}\right)\right| \\
&+\left|X\left(t_{i_{2}}^{(n)}\right)-X(t)\right| \\
& \leq \sigma(|| s-t \|) \varphi(\| s-t||)+2 c_{33}(p) G_{\sigma}(\sqrt{\log 1 /\|s-t\|}),
\end{aligned}
$$

because of $\left\|t-t_{i_{2}}^{(n)}\right\| \leq 3 \varepsilon_{n},\left\|t_{i_{1}}^{(n)}-t_{i_{2}}^{(n)}\right\| \leq\|s-t\|$ and (7.8). This completes the proof of Theorem 1. This theorem is true without concavity of $\sigma^{2}(x)$ and is also true under the condition $\left.\checkmark \overline{E\left[(X(s)-X(t))^{2}\right.}\right] \leq \sigma\left(|| s-\left.t\right|_{i}\right)$ instead of the equality. 
Remark 1 is easily obtained by setting

$$
\delta_{n}=\exp \left\{-H^{-1}\left(p^{n}\right)\right\} \quad \text { and } \quad \varphi(x)=\sqrt{(4+\varepsilon) H(\log 1 / x)}
$$

instead of (7.1). The last part of Remark 1 is obtained from the estimate $I_{n} \ll c_{34} \exp \left\{(1+\varepsilon) H\left(\log 1 / \delta_{n}\right)\right\} \quad(n \rightarrow \infty)$ by virtue of $(\text { A. } 1)^{(* *)}$.

Next we shall prove Theorem 2. Denote by $\left\{t_{t}^{(n)}, i=1,2, \cdots\right.$, $\left.M_{3 e_{n}}(D)\right\}$ a maximal $3 \varepsilon_{n}$-distinguishable set and choose $s_{i}^{(n)}$ such that $\left\|t_{i}^{(n)}-s_{i}^{(n)}\right\|=\varepsilon_{n}=2^{-n}$. Set

$$
\begin{aligned}
F_{n, i}=\left\{\omega ;\left|X\left(t_{i}^{(n)}\right)-X\left(s_{i}^{(n)}\right)\right|\right. & \leq \sigma\left(\left\|t_{i}^{(n)}-s_{i}^{(n)}\right\|\right) \\
& \left.\times \varphi\left(\left\|t_{i}^{(n)}-s_{i}^{(n)}\right\|\right)\right\},
\end{aligned}
$$

where $\varphi(x)=\sqrt{(1-\varepsilon) N \log 1 / x}$.

Setting

$$
\gamma_{i, j}^{(n)}=E\left[\left(X\left(t_{i}^{(n)}\right)-X\left(s_{i}^{(n)}\right)\right)\left(X\left(t_{j}^{(n)}\right)-X\left(s_{j}^{(n)}\right)\right)\right] / \sigma^{2}\left(\delta_{n}\right),
$$

we have

$$
\gamma_{i, j}^{(n)} \leq \sigma^{2}\left(\frac{4}{5} \varepsilon_{n}\right) / 2 \sigma^{2}\left(\varepsilon_{n}\right) \leq 1 / 2
$$

from Lemma 6, (i), Lemma 7, (i) and concavity of $\sigma^{2}(x)$. Now define auxiliary random variables $Z_{i}=\xi+\eta_{i} ; i=1,2, \cdots, M_{3 \mathrm{~s}_{n}}(D)$, where $\left\{\xi, \eta_{i}, i=1,2, \cdots\right\}$ are mutually independent Gaussian random variables with $E\left[\xi^{2}\right]=E\left[\eta^{2}\right]=1 / 2$ and $E[\xi]=E[\eta]=0$. Then by Lemma 4 , (v), Lemma 8 and choosing $\varepsilon^{\prime}$ such that $(1-\varepsilon)\left(1+\varepsilon^{\prime}\right)^{2}$ $<1$, we have

$$
\begin{aligned}
& \sum_{n}^{\infty} P\left(\bigcap_{i} F_{n, i}\right) \leq \sum_{n}^{\infty} P\left(Z_{i} \leq \varphi\left(\varepsilon_{n}\right) \quad \text { for any } i\right) \\
\leq & \sum_{n}^{\infty} P\left(\xi \leq-\varepsilon^{\prime} \varphi\left(\varepsilon_{n}\right)\right)+\sum_{n}^{\infty} P\left(\eta_{i} \leq\left(1+\varepsilon^{\prime}\right) \varphi\left(\varepsilon_{n}\right) \quad \text { for any } i\right) \\
<+\infty . &
\end{aligned}
$$

This implies that (4.7) holds with probability one, and if $\sigma(x)$ is a n.r.v.f., choosing $k \varepsilon_{n}$-distinguishable set such that $\gamma_{i, j}^{(n)} \ll \varepsilon^{\prime}$, we can set $\varphi(x)=\sqrt{(2-\varepsilon) N \log 1 / x}$ instead of (7.11). Remark 3 is obtained only by setting $\varphi(x)=\sqrt{(1-\varepsilon) H(\log 1 / x)}$ in the proof of Theorem 2 . 


\section{Proofs of Theorems 3 and 4}

First we shall prove Theorem 3. For the proof of the first part the following lemma plays an essential role. The idea of this lemma is due to T. Sirao [17].

Lemna 9. Let $(S, \rho)$ be a compact metric space satisfying $N_{\hat{\mathrm{a}}}(K) \leq c_{3 \mathrm{~s}}(d(K) / \varepsilon)^{N}$ for any compact subset $K$ of $S$, and let $\{X(s)$; $s \in S\}$ be a path continuous real valued G.p. with $E[X(s)]=0$ and $E\left[X(s)^{2}\right]=1$. Assume that there exists a non-decreasing cont nuous function $\sigma(x)$ such that $\sqrt{E\left[(X(s)-\bar{X}(t))^{2}\right]} \leq \sigma(\rho(s, t))$ and $F_{\sigma}(1)<+\infty$. Set

$$
A=\left\{\omega ; \sup _{s \in \mathcal{K}} X(s) \geq x+d_{8} F_{\sigma}(d(K))\right\}
$$

for any compact subset $K$ of $S$, where $d_{8}$ is a constant larger that $2^{5 / 2}(\sqrt{2}+1) \vee N$, then there exists a constant $d_{0}$ independent of $x$ and $K$ such that

$$
P(A) \leq d_{9} \Phi(x)
$$

holds for any

$$
0<x \sigma(d(K)) \leq d_{30},
$$

where $d_{10}$ is an arbitrary constant independent of $x$ and $K$.

Proof. Let $\left\{t_{i}^{(n)} ; i=1,2, \cdots, N_{s_{n}}(K)\right\}$ be minimal $\varepsilon_{n}$-net of $K$ and set

$$
\begin{aligned}
& \varepsilon_{n}=d(K) \exp \left\{-2^{n+1}\right\}, \quad x_{n}=d_{k}(\sqrt{2}-1) 2^{(n-1) / 2} \sigma\left(\varepsilon_{n-1}\right), \\
& A^{*}=\left\{\omega ; \sup _{s \in \kappa} X(s)>x+\sum_{k=1}^{\infty} x_{k}\right\}, \\
& A_{n}^{(1, j)}=\left\{\omega ; X\left(t_{i}^{(j)}\right)>x+\sum_{k=1}^{n} x_{k}\right\}, \\
& A_{n}=\bigcup_{\substack{1 \leq i \leq N_{g}(K) \\
0 \leq j \leq n}} A_{n}^{(i, j)}, \\
& A_{n}(\infty)=\left\{\omega ; \max _{\substack{1 \leq i \leq N_{k j}(\kappa) \\
0 \leq j \leq n}} X(t)>x+\sum_{k=1}^{\infty} x_{k}\right\} .
\end{aligned}
$$

Since $\sum_{k=1}^{\infty} x_{k}<d_{8} F_{\sigma}(d(K))$ and $A_{n}(\infty) \subset A_{n-1}(\infty) \subset \cdots$, $A^{*}=\bigcup_{n=1} A_{n}(\infty)$ and $A_{n}(\infty) \subset A_{n}$, we have 


$$
P(A) \leq P\left(A^{*}\right) \leq \lim _{n \rightarrow \infty} P\left(A_{n}\right) .
$$

Now we can estimate $P\left(A_{n}\right)$ as follows.

$$
\begin{aligned}
P\left(A_{n}\right) & \leq P\left(A_{n-1}\right)+P\left(A_{n} \cap A_{n-1}^{c}\right) \\
& \leq P\left(A_{n-1}\right)+\sum_{i=1}^{N} P\left(A_{n}^{(n, i)} \cap A_{n-1}^{c}\right) \\
& \leq P\left(A_{n-1}\right)+\sum_{i=1}^{N} P\left(A_{n}^{(n), i)} \cap A_{n-1}^{(n-1, m(i))^{c}}\right)
\end{aligned}
$$

where $m(i)$ is chosen such that $\rho\left(t_{i}^{(n)}, t_{m(i)}^{(n-1)}\right) \leq \varepsilon_{n-1}$.

Here we choose an auxiliary standard Gaussian random variable $Y$ independent of $X\left(t_{i}^{(n)}\right)$ such that $X\left(t_{m(1)}^{(n-1)}\right)=r X\left(t_{i}^{(n)}\right)+\sqrt{1-r^{2}} Y$, where $r=E\left[X\left(t_{i}^{(n)}\right) X\left(t_{m(1)}^{(n-1)}\right)\right]$. Since by definition

$$
\begin{aligned}
r & =1-\frac{1}{2} E\left[\left(X\left(t_{i}^{(n)}\right)-X\left(t_{m(t)}^{(n-1)}\right)\right)^{2}\right] \\
& \geq 1-\frac{1}{2} \sigma^{2}\left(\varepsilon_{n-1}\right) \equiv r_{0},
\end{aligned}
$$

it follows from Lemma 4, (iv) that

$$
\begin{aligned}
& P\left(A_{n}^{(n, i)} \cap A_{n-1}^{(n-1, m(i))}\right) \\
\leq & P\left(X\left(t_{t}^{(n)}\right)>x+\sum_{k=1}^{n} x_{k}, \quad r_{0} X\left(t_{i}^{(n)}\right)+\sqrt{1-r_{n}^{2}} \leq x+\sum_{k=1}^{n-1} x_{k}\right) \\
\leq & \Phi(x) \Phi\left(\left(1-r_{0}\right)\left(x+\sum_{k=1}^{n-1} x_{k}\right) / \sqrt{1-r_{0}^{2}}-r_{0} x_{n} / \sqrt{1-r_{0}^{2}}\right) .
\end{aligned}
$$

Hence for any $n \geq n_{0}=\min \left\{n: \sigma\left(\varepsilon_{n-1}\right) \leq 1\right\}$ we have

$$
\begin{aligned}
& \Phi\left(\left(1-r_{0}\right)\left(x+\sum_{k=1}^{n} x_{k}\right) / \sqrt{1-r_{0}^{2}}-r_{0} x_{n} / \sqrt{1-r_{0}^{2}}\right) \\
\leq & \Phi\left(x_{n} / 2 \sigma\left(\varepsilon_{n-1}\right)-x \sigma(d(K)) / 2-d_{8} F_{\sigma}(d(K)) / 2\right) \\
\leq & \Phi\left((\sqrt{2}-1) d_{8} 2^{(n-3) / 2}-d_{10} / 2-d_{8} F_{\sigma}(d(K)) / 2\right) \\
= & \Phi\left(P_{n}\right),
\end{aligned}
$$

where $P_{n}=(\sqrt{2}-1) d_{8} 2^{(n-3 / 2}-d_{10} / 2-d_{8} F_{\sigma}(d((K) / 2)$. Therefore combining (8.5), (8.7) and (8.8) we have

$$
\begin{aligned}
P\left(A_{n}\right) & \leq P\left(A_{n-1}\right)+N_{z_{n}}(K) \Phi\left(p_{n}\right) \Phi(x) \\
& \ldots \ldots \\
& \leq P\left(A_{n_{0}-1}\right)+\Phi(x) \sum_{k=n_{0}}^{n} N_{\xi k}(K) \Phi\left(p_{k}\right) .
\end{aligned}
$$

Since $P\left(A_{n_{0}-1}\right) \leq \Phi(x) \sum_{k=0}^{n_{i j}-1} N_{\mathrm{g} k}(K)$ and there exists a constant $d_{9}$ 
independent of $K$ such that

$$
\sum_{k \rightarrow 0}^{n_{0}-1} N_{\varepsilon k}(K)+\sum_{k=\pi_{0}}^{\infty} N_{\varepsilon k}(K) \Phi\left(p_{k}\right) \leq d_{0}<+\infty,
$$

we obtain the final result (8.2).

Using this lemma we shall prove the first part of Theorem 3. It is sufficient to show by Lemma 2, (iii) that $\phi \in \mathcal{V}^{\mu}(X)$ if $I_{u}(\sigma, \varphi)<+\infty$ under the assumption

$$
\sqrt{3 N \log 1 / x} \gg \phi \gg \sqrt{N \log 1 / x} .
$$

Let $S=(D \times D, \rho)$ be a direct product of $D$ with metric $\rho((t, s)$, $\left.\left(t^{\prime}, s^{\prime}\right)\right)=\sqrt{\left\|t-t^{\prime}\right\|^{2}+\left\|s-s^{\prime}\right\|^{2}},(t, s),\left(t^{\prime}, s^{\prime}\right) \in D \times D$ and $\left\{t_{i}^{(n)}, i=1,2\right.$, $\left.\cdots, N_{e n}(D)\right\}$ be a minimal $\varepsilon_{n}$-net of $D$. Set

$$
\begin{aligned}
& \varepsilon_{n}=\sigma^{-1}\left(\frac{\sigma\left(2^{-n}\right)}{\sqrt{n \log 2}}\right), \quad \delta_{n}=2^{-n-1}-2 \varepsilon_{n} \\
& I_{n}=\left\{(i, j) ; \delta_{n+1}-2 \varepsilon_{n} \leq\left\|t_{i}^{(n)}-t_{j}^{(n)}\right\| \leq \delta_{n}+2 \varepsilon_{n}\right\} \\
& D_{i}^{(n)}=\left\{s \in D ;\left\|t_{i}^{(n)}-s\right\| \leq \varepsilon_{n}\right\} \\
& S_{i . j}^{(n)}=D_{i}^{(n)} \times D_{j}^{(n)} \quad(i \neq j) \\
& Y(s, t)=\frac{X(s)-X(t)}{\sigma(\|s-t\|)}, \quad\left((s, t) \in S_{i, j}^{(n)}\right) \\
& E_{i, j}^{n}=\left\{\omega ; \sup _{(s, t) \in S_{i, j}^{(n)}} Y(s, t)>\varphi\left(2^{-n-1}\right)+2 d_{k} F_{\sigma}\left(4 \varepsilon_{n}\right) / \sigma\left(\delta_{n+1}-4 \varepsilon_{n}\right)\right\}
\end{aligned}
$$

Then it follows by concavity of $\sigma^{2}(x)$ that

$$
\begin{aligned}
& E\left[\left(Y(s, t)-Y\left(s^{\prime}, t^{\prime}\right)\right)^{2}\right] \\
& \leq \frac{2\left(\sigma^{2}\left(\left\|s-s^{\prime}\right\|\right)+\sigma^{2}\left(|| t-t^{\prime} \|\right)\right)}{\sigma(\| s-t \mid) \sigma\left(\left\|s^{\prime}-t^{\prime}\right\|\right)} \leq \frac{4 \sigma^{2}\left(\rho\left((s, t),\left(s^{\prime}, t^{\prime}\right)\right)\right)}{\sigma^{2}\left(\delta_{n-1}-4 \varepsilon_{n}\right)} .
\end{aligned}
$$

To apply Lemma 9 for $E_{s, j}^{n}$, we shall check the condition (8.3). Since $\lim _{n \rightarrow \infty} \varepsilon_{n} / \delta_{n-1}=0$ and $d\left(S_{i, j}^{(n)}\right) \leq 2 \sqrt{2} \varepsilon_{n}$, there exists an absolute constant $d_{11}$ such that

$$
2 \varphi\left(2^{-n-1}\right) \cdot \sigma\left(4 \varepsilon_{n}\right) / \sigma\left(\delta_{n+1}-4 \varepsilon_{n}\right) \leq d_{11}
$$

because of (8.11) and the property of n.r.v.f.. Hence using Lemma 9 we have

$$
P\left(E_{i, j}^{n}\right) \leq d_{12} \Phi\left(\varphi\left(2^{-n-1}\right)\right) .
$$


Since

(8. 16)

$$
\begin{aligned}
\# I_{n} & \leq c_{36}\left(\delta_{n}+2 \varepsilon_{n}\right)^{N} \varepsilon_{n}^{-2 N} \\
& \leq c_{37}\left(2^{-n}-2^{-n-1}\right) 2^{-(n+1)(N-1)} \varepsilon_{n}^{-2 N},
\end{aligned}
$$

we have

(8. 17)

$$
\begin{aligned}
& \sum_{n}^{\infty} \sum_{(i, j) \in I_{n}} P\left(E_{i, j}^{n}\right) \\
& \leq c_{38} \sum_{n}^{\infty} \frac{\left(2^{-n}-2^{-n-1}\right) 2^{-(n+1)<N-1)} \exp \left\{-\frac{1}{2} \phi^{2}\left(2^{-n-1}\right)\right\}}{\left.\sigma^{-1}\left(\sigma\left(2^{-n}\right) / \vee n \log 2\right)\right]^{2 N} \varphi\left(2^{-n-1}\right)} \\
& \leq c_{38} I_{u}(\sigma, \phi)<+\infty .
\end{aligned}
$$

As the same manner as (8.14) we have

$$
2 d_{8} F_{\sigma}\left(4 \varepsilon_{n}\right) \varphi\left(2^{-n-1}\right) / \sigma\left(\delta_{n+1}-4 \varepsilon_{n}\right) \leq c_{39} .
$$

This yields $\varphi+c_{s g} / \phi \in \mathcal{V}^{w}(X)$, but $I_{u}(\sigma, \varphi)<+\infty$ implies $I_{u}(\sigma, \varphi-$ $\left.c_{\triangleleft} / \varphi\right)<+\infty$. Hence we have $\varphi \in \mathcal{U}^{u}(X)$ if $I_{u}(\sigma, \varphi)<+\infty$.

Next we shall prove the last part of Theorem 3. For this purpose we shall use Lemma 3, (ii) and notice that it is sufficient to prove under the assumption (8.11) by Lemma 2, (iv). Let us assume that $I_{u}(\sigma, \varphi)=+\infty$ and let $\left\{t_{r}^{(n)} ; i=1,2, \cdots, M_{2 t n}(D)\right\}$ be a maximal $2 \varepsilon_{n}$-distinguishable set of $D$. Set

$$
\begin{gathered}
\varepsilon_{n}=\sigma^{-1}\left(\frac{\sigma\left(2^{-n-1}\right)}{\sqrt{(n-1) \log 2}}\right), \\
L_{n}=\left\{(i, j) ; 2^{-n} \leq\left\|t_{i}^{(n)}-t_{j}^{(m)}\right\| \leq 2^{-n-1}\right\},
\end{gathered}
$$

and

$$
F_{i, j}^{n}=\left\{\omega ; X\left(t_{i}^{(n)}\right)-X\left(t_{j}^{(n)}\right)>\sigma\left(\left\|t_{i}^{(n)}-t_{j}^{(n)}\right\|\right) \phi\left(\left\|t_{i}^{(n)}-t_{j}^{(n)}\right\|\right)\right\}
$$

for $(i, j) \in L_{n}$. Since we see

$$
P\left(F_{i, j}^{n}\right) \geq \frac{\exp \left\{-\frac{1}{2} \phi^{2}\left(2^{-\eta}\right)\right\}}{2 \sqrt{2 \pi} \phi\left(2^{-\eta}\right)}
$$

and

we have

$$
\sharp L_{n} \geq c_{41}\left(2^{-n}-2^{-n-1}\right) 2^{-n(N-1)} \varepsilon_{n}^{-2 N},
$$

(8. 20)

$$
\sum_{n}^{\infty} \sum_{(i, j) \in L_{n}} P\left(F_{i, j}^{n}\right) \geq
$$




$$
\begin{aligned}
& \geq c_{42} \sum_{n}^{\infty} \frac{\left(2^{-n}-2^{-n-1}\right) 2^{-x_{(N-1)}} \exp \left\{-\frac{1}{2} \varphi^{2}\left(2^{-n}\right)\right\}}{\varepsilon_{n}^{2 N} \varphi\left(2^{-n}\right)} \\
& \geq c_{42} I_{u}(\sigma, \varphi)=+\infty .
\end{aligned}
$$

To check the condition (b) of Lemma 3, the four point property plays an important role, which was pointed out by I. Kubo. Now we shall enumerate the events $\left\{F_{t, j}^{n}\right\}$ in some linear order $\left\{F^{(n)}\right\}_{h=1}$ such that $h>h^{\prime}$ if $\left\|t_{q}^{(n)}-t_{j}^{(n)}\right\| \geq\left\|t_{p}^{(m)}-t_{q}^{(m)}\right\|$, where $F^{(h)}\left(F^{\left(k^{\prime}\right)}\right)$ correspond to $F_{b, q}^{m}\left(F_{t, j}^{n}\right)$. Let us fix $(i, j)$ and sufficiently large $n$, and let us write for simplicity that

$$
\begin{aligned}
& \left\|t_{i}^{(m)}-t_{j}^{(m)}\right\|=r_{i, j}, \quad\left\|t_{p}^{(m)}-t_{q}^{(m)}\right\|=r_{p, q}, \quad\left\|t_{i}^{(n)}-t_{p}^{(m)}\right\|=r_{i, p}, \\
& \left\|t_{i}^{(n)}-t_{q}^{(m)}\right\|=r_{i, q},\left\|t_{j}^{(n)}-t_{p}^{(m)}\right\|=r_{j, p}, \\
& \text { and }\left\|t_{j}^{(m)}-t_{q}^{(m)}\right\|=r_{j, q} .
\end{aligned}
$$

Now set

(8.21) $\quad m_{0}=n+\frac{3 \log n}{\alpha \log 2} \quad(\alpha$ is the exponent of n.r.v.f. $\sigma(x))$,

$$
\begin{aligned}
& \gamma_{p, q}^{(m)}=E\left[\left(X\left(t_{i}^{(m)}\right)-X\left(t_{j}^{(m)}\right)\right)\left(X\left(t_{p}^{(m)}\right)-X\left(t_{q}^{(m)}\right)\right)\right] / \sigma\left(r_{i, j}\right) \sigma\left(r_{p, q}\right), \\
& L_{m, k}^{(1)}=\left\{(p, q) \in L_{m} ; 1-k / n \leq \gamma_{p, q}^{(m)} \leq 1-(k-1) / n, 1 \leq k \leq \sqrt{n}+1\right\}, \\
& L_{m, ~}^{(2)}=\left\{(p, q) \in L_{m} ; 1 / \varphi\left(r_{i, j}\right) \varphi\left(r_{p, q}\right) \leq \gamma_{p, q}^{(m)} \leq 1-1 / \sqrt{n}\right\} .
\end{aligned}
$$

Since we get by concavity of $\sigma^{2}(x)$

$$
\gamma_{p, q}^{(i)} \leq \sigma\left(r_{p, q} \wedge r_{i, q} \wedge r_{j, p}\right) / \sigma\left(r_{i, j}\right)
$$

combining (8.11), (8.22) and Lemma 5 we have

$$
\begin{aligned}
\gamma_{0, \emptyset}^{(m)} \varphi\left(r_{i, j}\right) \varphi\left(r_{p, q}\right) \leq \frac{\sigma\left(2^{-m_{0}-1}\right) \varphi\left(2^{-m_{0}-1}\right) \varphi\left(2^{-n+1}\right)}{\sigma\left(2^{-n}\right)} & \ll 1, \\
\text { if } \quad m & \geq m_{0} .
\end{aligned}
$$

Using Lemma 4, (ii) we have

$$
P\left(F_{i, j}^{n} \cap F_{p, 0}^{m}\right) \leq d_{3} P\left(F_{i, j}^{n}\right) P\left(F_{p, 8}^{m}\right)
$$

for any $m \geq m_{0}$. On the other hand if $(p, q) \in L_{m, k}^{(n)}$, the following are satisfied whose proofs are given below :

$$
\begin{gathered}
\left(r_{i, p}+r_{j, p}\right) \vee\left(r_{i, q}+r_{j, q}\right) \leq d_{13} r_{i, j}, \\
r_{\rho, q} \geq d_{14} r_{i, j},
\end{gathered}
$$




$$
\begin{gathered}
r_{i, q} \geq r_{j, q}, \quad r_{j, p} \geq r_{i, p}, \\
\text { (8.28) } m \leq n+d_{15}, \text { where } d_{15} \text { is independent of } n .
\end{gathered}
$$

By Lemma (6), (i) and (8.22) we have

$$
\begin{aligned}
\gamma_{p, q}^{(m)} & \leq \frac{\sigma^{2}\left(\left|r_{i, q}+r_{j, p}-r_{i, p}-r_{j, q}\right|\right)}{2 \sigma\left(r_{i, j}\right) \sigma\left(r_{p, q}\right)} \\
& \leq \frac{\sigma^{2}\left(4 r_{i, j} r_{p, q} / R\right)}{2 \sigma\left(r_{i, j}\right) \sigma\left(r_{p, q}\right)},
\end{aligned}
$$

where $R=\left(r_{i, p}+r_{j, p}\right) \vee\left(r_{i, q}+r_{j, q}\right)$. This implies (8.25). It follows (8.26) from

$$
\sqrt{3} / 2 \leq \gamma_{j, q}^{(m)} \leq \frac{\sigma\left(r_{p, q}\right)}{\sigma\left(r_{i, j}\right)}
$$

Analogously we have

$$
\sqrt{3} / 2 \leq \gamma_{p, q}^{(m)} \leq \frac{\sigma^{2}\left(r_{i, q}\right)+\sigma^{2}\left(r_{i, j}\right)-\sigma^{2}\left(r_{j, q}\right)}{\sqrt{3}}
$$

which yields (8.27). It follows (8.28) from (8.26).

Now we shall estimate $\sharp L_{m, k}^{(1)}$ for $n \leq m \leq n+d_{15}, 1 \leq k \leq \sqrt{ } n+1$. For this end we divide following four cases.

Case 1. $r_{i, q} \vee r_{j, p} \leq r_{i, j}$. Using concavity of $\sigma^{2}(x)$ we have

$$
\begin{aligned}
\gamma_{p, Q}^{(m)} & \leq \frac{\sigma^{2}\left(r_{i, q}\right)+\sigma^{2}\left(r_{p, q}\right)-\sigma^{2}\left(r_{1,0}\right)}{2 \sigma\left(r_{p, q}\right) \sigma\left(r_{i, q}\right)} \\
& \leq 1-\sigma^{2}\left(r_{i, 0}\right)-\sigma^{2}\left(r_{p, q}\right)\left(\sqrt{1+\sigma^{2}\left(r_{i, p}\right) / \sigma^{2}\left(r_{p, q}\right)}-1\right)^{2} \\
2 \sigma\left(r_{p, q}\right) & \sigma\left(r_{i, j}\right)
\end{aligned}
$$

Since combining (8.26) and $r_{i, p} \leq r_{j, p} \leq r_{i, j}$ we have

$$
\begin{aligned}
\sqrt{1+\sigma^{2}\left(r_{i, p}\right) / \sigma^{2}\left(r_{p, q}\right)}-1 & \leq c_{i 3} \sigma\left(r_{i, p}\right) / \sigma\left(r_{p, q}\right) \\
& \left(0<c_{i 3}<1\right),
\end{aligned}
$$

there exists a constant $c_{14}>0$ such that

$$
\gamma_{p, 0}^{(m)} \leq 1-c_{14} \sigma^{2}\left(r_{i, p}\right) / \sigma^{2}\left(r_{i, j}\right) .
$$

As the same manner as (8.34) we get

$$
\gamma_{p, q}^{(m)} \leq 1-c_{45} \sigma^{2}\left(r_{j, q}\right) / \sigma^{2}\left(r_{i, j}\right) .
$$

Case 2. $r_{i, q} \wedge r_{j, p} \geq r_{i, j}$. First we shall assume that $r_{i, p} \leq r_{j, q}$. 
By concavity of $\sigma^{2}(x)$ and Lemma 6 , (ii) we have

$$
\begin{aligned}
\gamma_{p, q}^{(m)} & \leq \frac{\sigma^{2}\left(r_{i, q}\right)+\sigma^{2}\left(r_{j, p}\right)-\sigma^{2}\left(r_{i, p}\right)-\sigma^{2}\left(r_{j, q}\right)}{2 \sigma^{2}\left(r_{p, q}\right)} \\
& \leq 1-\frac{\sigma^{2}\left(r_{i, p}\right)+\sigma^{2}\left(r_{j, q}\right)-\sigma^{2}\left(r_{i, q}\right)}{2 \sigma^{2}\left(r_{p, q}\right)}-\sigma^{2}\left(r_{j, p}\right)+2 \sigma^{2}\left(r_{p, q}\right) \\
& \leq 1-\frac{\sigma^{2}\left(r_{i, p}+r_{p, q}-r_{j, p}\right)+\sigma^{2}\left(r_{j, q}+r_{p, q}-r_{i, q}\right)}{2 \sigma^{2}\left(r_{i, j}\right)}
\end{aligned}
$$

We shall show that it is imposible to find $d_{16}<\frac{d_{14}}{1+d_{13}}$ such that

$$
r_{i, p}+r_{p . q}-r_{j, p} \leq d_{16} r_{i, p}
$$

and

$$
r_{j, q}+r_{p, q}-r_{i, q} \leq d_{16} r_{i, p} .
$$

Because, combining (8.37), (8.48) and from Lemma 7, (ii) we have

$$
\begin{aligned}
2\left(r_{p, q}-d_{16} r_{i, p}\right) & \leq r_{i, q}+r_{j, p}-r_{j, q}-r_{i, p} \\
& \leq \frac{2 r_{i, j} r_{p, q}}{r_{i, j}+r_{i, p}} .
\end{aligned}
$$

This implies by $(8.25)$ that

$$
\begin{aligned}
r_{p, q} & \leq d_{16}\left(r_{i, j}+r_{i, p}\right) \\
& \leq d_{16}\left(1+d_{13}\right) r_{i, j}<d_{14} r_{i, j},
\end{aligned}
$$

which contradicts to $(8.26)$. Therefore we have

$$
\gamma_{p, \eta}^{(m)} \leq 1-\frac{\sigma^{2}\left(d_{16} r_{i, p}\right)}{2 \sigma^{2}\left(r_{i, j}\right)}
$$

To estimate $r_{j, q}$, it needs to get the sharper inequality than (8. 36). That is, we have

$$
\begin{aligned}
\gamma_{p, q}^{(m)} & \leq 1-\frac{1}{2 \sigma\left(r_{i, j}\right)} \frac{\sigma\left(r_{p, q}\right)}{2}\left\{\sigma^{2}\left(r_{i, p}\right)+\sigma^{2}\left(r_{p, q}\right)-\sigma^{2}\left(r_{i, q}\right)\right. \\
& \left.+\sigma^{2}\left(r_{j, q}\right)+\sigma^{2}\left(r_{i, j}\right)-\sigma^{2}\left(r_{j, p}\right)-\left(\sigma\left(r_{i, j}\right)-\sigma\left(r_{p, q}\right)\right)^{2}\right\} .
\end{aligned}
$$

Using concavity of $\sigma^{2}(x)$ and Lemma 6, (ii) we have (8. 43) $\sigma^{2}\left(r_{i, p}\right)+\sigma^{2}\left(r_{p, q}\right)-\sigma^{2}\left(r_{i, q}\right) \geq \sigma^{2}\left(r_{p, q}\right)-\sigma^{2}\left(r_{i, q}-r_{i, p}\right) \geq 0$, 


$$
\sigma^{2}\left(r_{j, q}\right)+\sigma^{2}\left(r_{i, j}\right)-\sigma^{2}\left(r_{j, p}\right) \geq \sigma^{2}\left(r_{j, p}+r_{i, j}-r_{j, p}\right)
$$

and

$$
\begin{aligned}
\left(\sigma\left(r_{i, j}\right)-\sigma\left(r_{p, q}\right)\right)^{2} & \leq \sigma^{2}\left(r_{p, q}\right)\left(\sqrt{1+\frac{\sigma^{2}\left(r_{i, q}-r_{p, q}\right)}{\sigma^{2}\left(r_{p, q}\right)}}-1\right)^{2} \\
& \leq c_{46} \sigma^{2}\left(r_{i, j}-r_{p, q}\right) \quad\left(c_{46}<1\right)
\end{aligned}
$$

Hence combining (8.42), (8.43). (8.44) and (8.45) we have

$$
\begin{aligned}
\gamma_{p, 4}^{(m)} & \leq 1-\frac{\sigma^{2}\left(r_{j, q}+r_{i, j}-r_{j, p}\right)-c_{46} \sigma^{2}\left(r_{i, j}-r_{p, q}\right)}{2 \sigma^{2}\left(r_{i, j}\right)} \\
& \leq 1-\frac{\left(1-c_{86}\right) \sigma^{2}\left(r_{j, q}+r_{i, j}-r_{j, p}\right)}{2 \sigma^{2}\left(r_{i, j}\right)} \\
& \leq 1-\frac{\left(1-c_{66}\right) \sigma^{2}\left(r_{j, q}-r_{i, p}\right)}{2 \sigma^{2}\left(r_{i, j}\right)}, \quad \text { if } \quad r_{j, q} \geq 2 r_{i, p} .
\end{aligned}
$$

If $r_{i, p}>r_{j, q}$, we have the same estimate as (8.41) and (8.46) by substitution of $(i, p)$ and $(j, q)$.

Case 3. $r_{i, q} \leq r_{i, j} \leq r_{j, p}$. We notice that (8.34) and (8.46) are still valid without any change.

Case 4. $r_{j, p} \leq r_{i, j} \leq r_{i, q}$. This case is reduced to Case 3 by substitution of $(i, q)$ and $(j, p)$.

Therefore through the all case it follows from Lemma 8 and Lemma 5 that

$$
\# L_{m, k}^{(1)} \leq c_{47}\left(\sigma^{-1}\left(c_{48} \sqrt{k / n} \sigma\left(2^{-n}\right)\right)\right)^{2 N} \varepsilon_{m}^{-2 N} \leq c_{49} k^{c_{50}} .
$$

Next we shall estimate $\$ L_{7 t}^{(2)}\left(m \leq m_{0}\right)$. It follows that

$$
R \leq r_{i, j}\left(\log 1 / r_{i, j}\right)^{2 / \infty},
$$

where $R$ is defined in (8.29). Because, if $(p, q) \in L_{n}^{(2)}, m \leq m_{0}$ then by $(8.29)$ we have

$$
\gamma_{p, 4}^{(m)} \sqrt{n m} \leq c_{\mathrm{s} 1} \frac{\sigma^{2}\left(4 r_{\mathrm{p}, 9}\left(\log 1 / r_{i, g}\right)^{-2 / \infty}\right)}{\sigma^{2}\left(r_{p, q}\right)} \sqrt{n m} \ll 1
$$

Therefore we have

(8. 49) $L_{i m}^{(2)} \leq c_{52}\left(r_{i, j}\left(\log 1 / r_{i, j}\right)^{2 / \alpha}\right)^{2 N} \varepsilon_{m}^{-2 N} \leq c_{53} n^{c_{54}}$.

Hence it follows by (8.11), (8.47), (8.49) and Lemma 4, (iii) that 


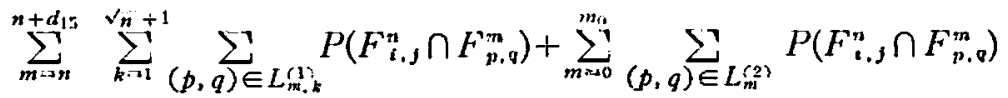

$$
\begin{aligned}
& \leq\left[d_{15} \sum_{k=1}^{\infty} c_{45} k^{r} 50 \exp \left\{-c_{55} k\right\}+c_{53} n^{c_{54}} \exp \left\{-c_{56} \sqrt{n}\right\}\right] P\left(F_{1 . j}^{n}\right) \\
& \leq c_{57} P\left(F_{i, j}^{n}\right) \text {. }
\end{aligned}
$$

Using Lemma 3 , we have

$$
P\left(\overline{\lim } F^{(h)}\right) \geq 1 / d_{3} .
$$

On the other hand we notice that by setting

$$
\psi(x)=G^{-1}\left[G(\varphi(x))+\log \int_{x} \frac{u^{N^{-1}} \exp \left\{-\frac{1}{2} \phi^{2}(u)\right\}}{\left[\sigma^{-1}\left(\frac{\sigma(u)}{\sqrt{\log 1 / u}}\right)\right]^{2 N} \varphi(u)} d u\right],
$$

where $G(x)=\frac{1}{2} x^{2}+\log x$, we get $I_{u}(\sigma, \psi)=+\infty$ and $\lim _{0 \nmid x} \psi^{2}(x)-\varphi^{2}(x)$ $=+\infty$. Therefore by (8.50), Lemma 1 and concavity of $\sigma^{2}(x)$, it

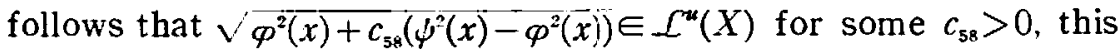
yields $\varphi(x) \in \mathcal{L}^{u}(X)$. Thus we get the proof of the last half of Theorem 3 .

The first part of Corollary 3 is an immediate consequence of Theorem 3. To prove the last part of Corollary 3, we set $f(x)=\int_{0}^{x}\left(\alpha-a\left(e^{-u}\right)\right) d u$. Since we can wright

$$
\sigma_{2}(x)=c(x) \exp \{-f(\log 1 / x)\},
$$

where $c(x)$ is a continuous functions such that $c_{59} \geq c(x) \geq c_{60}>0$, we have

$$
\begin{aligned}
& \sigma_{2}^{-1}\left(\frac{\sigma_{2}(x)}{\log 1 / x}\right)=\exp \left\{-f^{-1}\left(f(\log 1 / x)+\frac{1}{2} \log _{(2)} 1 / x+c^{*}(x)\right)\right\} \\
& \asymp \exp \left\{-\left(\log 1 / x+\frac{\log _{(2)} 1 / x}{2 \alpha}\right)\right\} \exp \left\{\frac{a(x) \log _{(2)} 1 / x}{\alpha(\alpha-a(x))}\right\} \\
& \asymp \sigma_{1}^{-1}\left(\frac{\sigma_{1}(x)}{\sqrt{\log 1 / x}}\right),
\end{aligned}
$$

if $\left|a(x) \log _{i_{2}} 1 / x\right| \leq c_{17}$, which proves the last part of Corollary 3 . Corollary 4 follows from the fact that there exists $x_{0}$ such that

$$
\begin{aligned}
& f^{-1}\left(f(\log 1 / x)+\frac{1}{2} \log _{(x)} 1 / x+c^{*}(x)\right) \\
= & \log 1 / x+\frac{\log _{(2)} 1 / x+2 c^{*}(x)}{2\left(\alpha-a\left(x_{0}\right)\right)},
\end{aligned}
$$


where, $\log 1 / x \leq x_{0} \leq f^{-1}\left(f(\log 1 / x)+\frac{1}{2} \log _{(2)} 1 / x+c(x)\right)$, and that we can chose $c_{61}$ by virtue of (4.14) such that

$$
\begin{aligned}
& I_{u}\left(\sigma_{1}, \varphi\right)<+\infty, \quad I_{u}\left(\sigma_{2}, \varphi\right)=+\infty \text { or } I_{u}\left(\sigma_{1}, \varphi\right)=+\infty, \\
& I_{u}\left(\sigma_{2}, \varphi\right)<+\infty \text { for }
\end{aligned}
$$

(8. 55) $\varphi(x)=\sqrt{2 N \log 1 / x+\left(\frac{2 N}{\alpha}+1\right) \log _{(2)} 1 / x+c_{61}} \overline{\log _{(3)} 1 / x}$.

Finally we shall prove Theorem 4. The proof of this theorem is essentially based on the same method as that of Theorem 3 . So we shall use the same notation and denote by $\left({ }^{\circ}\right)$, or $\left({ }^{\prime}\right)$ if we need slightly modified notations. Set

$$
\begin{gathered}
h(x)=\int_{0}^{x} a\left(e^{-u}\right) d u, \\
\tilde{\varepsilon}_{n}=\exp \left\{-h^{-1}\left(h(n \log 2) \div \frac{1}{2} \log (n \log 2)\right)\right\}, \\
\varphi_{\varepsilon}(x)=\sqrt{2 N \log } \overline{1} / x+2 \overline{N(1+\varepsilon)\left(\log _{(2)} 1 / x\right) / \overline{a(x)}} .
\end{gathered}
$$

Since by the condition (i) it follows that

$$
\begin{gathered}
h^{-1}\left(h(\log 1 / x)+\frac{1}{2} \log _{(2)} 1 / x+c_{2^{2}}\right) \\
\ll \log 1 / x+\frac{\left(1+\varepsilon^{\prime}\right) \log _{(2)} 1 / x}{2 a(x)}
\end{gathered}
$$

for any $\varepsilon>\varepsilon^{\prime}>0$, we have $\varphi_{\varepsilon}\left(2^{-n-1}\right) \sigma\left(4 \bar{\varepsilon}_{n}\right) / \sigma\left(\delta_{n+1}-4 \tilde{\varepsilon}_{n}\right) \leq d_{1 \bar{\tau}}$. Hence using Lemma 9 we get

$$
P\left(E_{i, j}^{n}\right) \leq d_{18} \Phi\left(\phi_{i}\left(2^{-n-1}\right)\right) .
$$

Since

$$
\sharp I_{n} \leq c_{63}\left(2^{-n}-2^{-n-1}\right) 2^{\left.-(n+1) \times N^{-1}\right)} \tilde{\mathcal{E}}_{n}^{-2 N}
$$

it follows that

$$
\begin{aligned}
& \sum_{n}^{\infty} \sum_{(i, j) \in \boldsymbol{J}_{n}} P\left(E_{i, j}^{n}\right) \leq c_{64} \sum_{n}^{\infty}\left(2^{-n}-2^{-n-1}\right) 2^{-(n+1)(N-1)} \\
& \times \exp \left\{2 N\left(\log 2^{n}+\frac{1+\varepsilon^{\prime}}{2} \frac{\log \left(2,2^{n}\right.}{a\left(2^{-\eta}\right)}-\frac{1}{2} \varphi_{\varepsilon}^{2}\left(2^{-n-1}\right)\right)\right\} / \varphi_{\varepsilon}\left(2^{-n-1}\right) \\
& <+\infty .
\end{aligned}
$$


While from Lemma 11 we have

$$
\frac{F_{\sigma}\left(4 \tilde{\varepsilon}_{n}\right) \varphi_{\mathrm{g}}\left(2^{-n-1}\right)}{\sigma\left(\delta_{n+1}-4 \tilde{\varepsilon}_{n}\right)} \leq \frac{c_{65}}{\sqrt{a\left(2^{-n-1}\right)}}
$$

This yields $\varphi_{\mathrm{g}}(x)+c_{66} /\left(\varphi_{\mathrm{g}}(x) \sqrt{a(x)}\right) \ll \varphi_{2 \mathrm{~g}} \in \mathcal{U}^{u}(X)$, for any $\varepsilon>0$, then the first part of Theorem 4 was proved.

Next we shall prove the last part of Theorem 4. Set

$$
\varepsilon_{n}^{\prime}=\exp \left\{-h^{-1}\left(h(n \log 2)+\frac{1+\varepsilon}{2} \log (n \log 2)\right)\right\} \quad(\varepsilon<0) \text {. }
$$

Since by the condition (i) it follows that

$$
\begin{gathered}
\exp \left\{-\left(n \log 2+\frac{(1+\varepsilon) \log (n \log 2)}{2 a\left(2^{-n}\right)}\right)\right. \\
\gg \varepsilon_{n}^{\prime} \gg \exp \left\{-\left(n \log 2+\frac{(1-\varepsilon) \log (n \log 2)}{2 a\left(2^{-n}\right)}\right)\right\} \quad(\varepsilon<0),
\end{gathered}
$$

we get

(8. 62)

$$
\begin{aligned}
& \sum_{n}^{\infty} \sum_{\left(i, j, j \in L_{i n}\right.} P\left(F_{i, s}^{n}\right) \\
\geq & c_{0 ;} \sum_{n}^{\infty}\left(2^{-n}-2^{-n-1}\right) 2^{-n(N-1)} \exp \left\{2 N\left(\log 2^{n}+\frac{1+\varepsilon}{2} \frac{\log _{(2)} 2^{n}}{a\left(2^{-n}\right)}\right)\right\} \\
\times & \exp \left\{-\frac{1}{2} \varphi_{\mathrm{\varepsilon}}^{2}\left(2^{-n-1}\right)\right\} / \varphi_{\varepsilon}\left(2^{-n}\right)=+\infty \quad(\varepsilon<0) .
\end{aligned}
$$

To check the condition (b) of Lemma 3, we shall follow step by step the last part of the proof of Theorem 3. Set

$$
\begin{aligned}
& h(x, y)=\int_{x}^{y} a\left(e^{-u}\right) d u, \\
& \check{m}_{0}=n+\frac{2 \log n}{a\left(2^{-n}\right) \log 2} .
\end{aligned}
$$

Since by the condition (ii) we get

$$
\log _{a(t)} \frac{1 / x}{x} \ll-\varepsilon \log 1 / x \quad(\varepsilon<0)
$$

it follows analogously in (8.23) that

$$
\begin{aligned}
& \gamma_{p . q}^{(m)} \varphi_{\varepsilon}\left(r_{i, j}\right) \varphi_{\varepsilon}\left(r_{p . q}\right) \\
& \ll c_{69} \sigma\left(2^{-m-1}\right) 、 m n / \sigma\left(2^{-n}\right)
\end{aligned}
$$




$$
\begin{aligned}
& \ll c_{69} n \exp \left\{-h\left(n \log 2, n \log 2+\frac{2 \log n}{a\left(2^{-n}\right)}\right)\right\} \\
& \ll 1, \quad \text { if } \quad m \geq \tilde{m}_{0} .
\end{aligned}
$$

By virtue of (8.61), we have

$$
\begin{aligned}
& \quad L_{m, k}^{(1)} \leq c_{70}\left(\sigma^{-1}\left(c_{71} \vee \bar{k} / n \sigma\left(2^{-n}\right) / \varepsilon_{m}^{\prime}\right)\right)^{2 N} \\
\leq & c_{72} \exp \left\{N(1+\varepsilon) \log k / a\left(2^{-n}\right)+N \varepsilon \log (n \log 2) / 2 a\left(2^{-n}\right)\right\}
\end{aligned}
$$

for $1 \leq k \leq n^{1-\beta / 2}+1$, ( $\beta$ is defined in Condition (ii)). Therefore we get

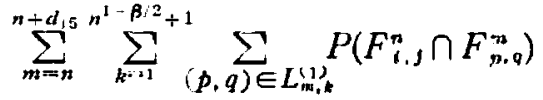

$$
\begin{aligned}
& \leq c_{73} \sum_{k=1}^{\infty} \exp \left\{N(1+\varepsilon) \log k / a\left(2^{-n}\right)+N \varepsilon \log (n \log 2) / 2 a\left(2^{-n}\right)-c_{74} k\right\} \\
& \leq d_{19} P\left(F_{i .1}^{n}\right) \quad(\varepsilon<0) \text {. } \\
& \times P\left(F_{i, j}^{n}\right)
\end{aligned}
$$

While if $R=\left(r_{i, p}+r_{j, p}\right) \vee\left(r_{i, p}+r_{j, q}\right) \geq 4 \exp \left\{-n \log 2+\frac{c_{i 5} \log n}{a\left(2^{-n}\right)}\right\}$ and $m \leq m_{0}$, it follows by (8.29) that

$$
\begin{aligned}
& \gamma_{p, Q}^{(n)} \varphi_{\mathrm{e}}\left(r_{i, j}\right) \varphi_{\mathrm{z}}\left(r_{p, q}\right) \\
\leq & \sigma\left(2^{-n-c_{75} \log n / a\left(2^{-n}\right)}\right) \varphi_{\mathrm{g}}\left(2^{-n-1}\right) \varphi_{\mathrm{e}}\left(2^{-m_{0}-1}\right) / 2 \sigma\left(2^{-n}\right) \\
\leq & \left.c_{16} n \exp \left\{-h\left(\left(n+c_{75} \log n / a\left(2^{-n}\right)\right) \log 2\right)-h(n \log 2)\right)\right\} \\
\ll & 1
\end{aligned}
$$

for a suitably chosen constant $c_{75}$, and we have

$$
\begin{aligned}
\# \tilde{L}^{(2)} & \leq c_{77}\left(\exp \left\{-n \log 2+c_{75} \log n / a\left(2^{-n}\right)\right\} / \varepsilon_{m_{0}}^{\prime}\right)^{2 N} \\
& \leq c_{78} \exp \left\{c_{79} \log n / a\left(2^{-n}\right)\right\} \\
& \leq c_{78} \exp \left\{c_{80} n^{1-\beta} \log n\right\} .
\end{aligned}
$$

Accordingly we get by Lemma 4, (iii),

$$
\text { (8. 70) } \begin{aligned}
& \sum_{m=3 n}^{\tilde{n}_{0}} \sum_{(p, q) \in \tilde{L}_{m}^{(2)}} P\left(F_{i, j}^{n} \cap F_{p, q}^{n}\right) \\
\leq & \left\{c_{78} 2 \log n \exp \left\{c_{80} n^{1-\beta} \log n-c_{81} n^{1-\beta / 2}\right\} / a\left(2^{-n}\right) \log 2\right\} P\left(F_{i, j}^{n}\right) \\
\leq & d_{21} P\left(F_{i, j}^{n}\right) .
\end{aligned}
$$

Hence combining (8.62), (8.67) and (8.70) we have $\phi_{2 z} \in \mathcal{L}^{u}(X)$ for $\varepsilon<0$. This completes the proof of Theorem 4 . 


\section{Proofs of Theorems 5 and 6}

The proofs of Theorems 5 and 6 are based on those of M. B. Marcus' theorems [13]. To prove Theorem 5 we shall use the following Lemma due to $X$. Fernique. Let $(S, \rho)$ be a compact metric space such that

$$
N_{\bar{z}}(K) \leq d_{22}\left(\frac{d(K)}{\varepsilon}\right)^{N}
$$

for any compact subset $K$ of $S$, and $\{X(s) ; s \in S\}$ be a path continuous $G . p$. with $E[X(s)]=0$ such that $E\left[(X(s)-X(t))^{2}\right] \leq \sigma^{2}(\rho(s, t)$ ), where $\sigma(x)$ is a non-decreasing continuous function satisfying $F_{\sigma}(1)<+\infty$.

\section{Lemma 10. [5]}

$$
P\left(\sup _{s \in K}|X(s)| \geq x\left(\left.|| \Gamma\right|_{K}+4 F_{\sigma}(d(K)) / \sqrt{\log p}\right)\right) \leq 9 d_{22} p^{2 N} \Phi(x)
$$

for any $p>1$ and $x \geq \sqrt{1+4 \bar{N} \log p}$; where $\|\Gamma\|_{K}=\sup _{s, l \in K}|E[X(s) X(t)]|$.

Now we shall prove Theorem 5 . Set

$$
\begin{aligned}
& q(x)=\sqrt{\left.2\left\{\left(\log _{(2)} 1 / x\right) \vee F_{\sigma}(x) / \sigma(x)\right)\right\}}, \\
& \delta_{n}=e^{-n}, S_{n}=\{s \in S ; \\
& Y(s)=\frac{X(s)-X\left(s_{0}\right)}{\sigma\left(\left\|s-s_{0}\right\|\right)}, \quad s \in S_{n} .
\end{aligned}
$$

Since we get

$$
\begin{aligned}
& E\left[Y(s)^{2}\right]=1, \\
& E\left[(Y(s)-Y(t))^{2}\right] \\
& \quad \leq \frac{\sigma^{2}(\| s-t||)}{\sigma\left(\left\|s-s_{0}\right\|\right) \sigma\left(\left\|t-s_{0}\right\|\right)} \\
& \quad \leq \sigma^{2}(\| s-t||) / \sigma^{2}\left(\hat{\delta}_{n+1}\right) \quad \text { for } s, t \in S_{n},
\end{aligned}
$$

using Lemma 10 for $p^{q}=n$ it follows that

$$
\begin{gathered}
P\left(\sup _{s \in S_{x}}|Y(s)| \geq c_{\mathrm{B} 2} \rho\left(\delta_{n}\right)\right) \\
\leq 9 d_{22} n^{2 N / 9} \Phi\left(x_{n}\right),
\end{gathered}
$$

where 


$$
x_{n}=\frac{c_{\mathrm{g} 2} \sqrt{\log n} \varphi\left(\delta_{n}\right)}{\sqrt{\log n+4 \sqrt{q} F_{\sigma}\left(2 \delta_{n}\right) / \sigma\left(\delta_{n+1}\right)}} .
$$

By the definition of $\phi$ we have

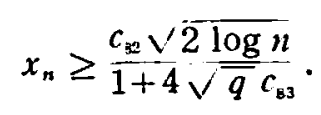

Hence for suitably chosen $q, c_{\mathrm{Bz}}$ and $c_{83}$ we have

$$
\sum_{n}^{\infty} P\left(\sup _{i \in \mathcal{S}_{n}} \mid Y(s)_{i} \geq c_{\mathrm{g} 2} \varphi\left(\delta_{n}\right)\right)<+\infty .
$$

In particular in case of (i) we can set $c_{\mathrm{gz}}$ arbitrarily closed to 1 because $c_{\mathrm{B} 3}$ can be chosen arbitrarily small for large $n$. The proof follows from Lemma 12 and Lemma 3.

Next we shall prove (i) of Theorem 6 . Set

$$
\begin{aligned}
& \varphi_{\mathrm{z}}(x)=\sqrt{ }(2-\varepsilon) \overline{\log _{(2)}} \overline{1 / x}, \\
& \|e\|=1, s^{n}=s_{0}+2^{-n} e \in D \\
& \gamma_{n, m}=E\left[\left(X\left(s_{n}\right)-X\left(s_{\mathrm{t}}\right)\right)\left(X\left(s_{m}\right)-X\left(s_{0}\right)\right)\right] / \sigma\left(2^{-n}\right) \sigma\left(2^{-m}\right) \\
& F^{n}=\left\{\omega ;\left|X\left(s_{n}\right)-X\left(s_{0}\right)\right| \geq \sigma\left(2^{-n}\right) \varphi_{\varepsilon}\left(2^{-n}\right)\right\} .
\end{aligned}
$$

Then we have

$$
\begin{aligned}
\sum_{n}^{\infty} P\left(F^{n}\right) & =\sum_{n}^{\infty} \Phi\left(\varphi_{s}\left(2^{-n}\right)\right) \\
& =\sum_{n}^{\infty} \Phi(\sqrt{(2-\varepsilon) \overline{\log }(\overline{n \log 2})})=+\infty .
\end{aligned}
$$

Since it follows that

(9. 7) $\gamma_{n, m}=\frac{\sigma^{2}\left(2^{-n}\right)+\sigma^{2}\left(2^{-m}\right)-\sigma^{2}\left(2^{-n}-2^{-m}\right)}{2 \sigma\left(2^{-n}\right) \sigma\left(2^{-m}\right)} \leq \sigma\left(2^{-m}\right) / \sigma\left(2^{-n}\right)$,

we have

$$
\begin{aligned}
& \gamma_{n, m} \varphi_{\mathrm{e}}\left(2^{-n}\right) \varphi_{\mathrm{z}}\left(2^{-m}\right) \\
& \leq \sigma\left(2^{-m}\right)(2-\varepsilon) \sqrt{\log (n \log 2)} \log \left(m \log \overline{2)} / \sigma\left(2^{-n}\right)\right. \\
& \ll 1 \text { for } m \geq m_{\mathrm{s}} \text {. }
\end{aligned}
$$

This follows from Lemma 4 , (i1) that

$$
P\left(F^{n} \cap F^{m}\right) \leq d_{3} P\left(F^{n}\right) P\left(F^{m}\right) .
$$

Since there exists a constant $c_{84}$ such that $\gamma_{n, m} \leq \sigma\left(2^{-m}\right) / \sigma\left(2^{-n}\right)$ 
$\leq 1 / 2$ for $m \geq n+c_{s t}$, we have

(9.9)

$$
\begin{aligned}
& \left(\sum_{m=n}^{n+c_{84}}+\sum_{n+c_{84}}^{m_{0}}\right) P\left(F^{n} \cap F^{m}\right) \\
\leq & c_{84} P\left(F^{n} \cap F^{m}\right)+\frac{3 \log n}{\alpha \log 2} \exp \left\{-c_{\mathrm{ks}} \log n\right\} P\left(F^{n}\right) \\
\leq & d_{23} P\left(F^{n}\right) .
\end{aligned}
$$

Combining (9.6), (9.8) and (9.9) we get Theorem 6, (i). To prove Theorem 6, (ii) set

$$
\begin{aligned}
& \varphi(x)=\sqrt{1 / a(x)}, \quad r_{n}=\inf \{r ; a(r)=1 / \log n\} \\
& F^{n}=\left\{\omega ; X\left(s_{0}+r_{n} e\right)-X\left(s_{0}+r_{n} e\right) \geq c_{86} \sigma\left(r_{n}\right) \varphi\left(r_{n}\right)\right\} .
\end{aligned}
$$

Then we have

$$
P\left(F^{n}\right)=\Phi\left(\frac{c_{86} \sigma\left(r_{n}\right) \varphi\left(r_{n}\right)}{\sigma\left(r_{n}-r_{n+1}\right)}\right) .
$$

By the definition of $r_{n}$ and (5.7), we get

This follows

$$
\begin{aligned}
\frac{1}{\log n}-\frac{1}{\log (n+1)} & =a\left(r_{n}\right)-a\left(r_{n+1}\right) \\
& \leq c_{24} \frac{r_{n}-r_{n+1}}{r_{n+1}} a\left(r_{n+1}\right) \\
& \leq \frac{c_{24}}{\log (n+1)} \frac{r_{n}-r_{n+1}}{r_{n+1}} .
\end{aligned}
$$

$$
\frac{r_{n+1}}{r_{n}-r_{n+1}} \leq 2 c_{24} n \log n .
$$

While if $r_{n+1} \leq r_{n} / 2$ we have

$$
\frac{\sigma^{2}\left(r_{n}\right)}{\sigma^{2}\left(r_{n}-r_{n-1}\right)} \leq \frac{\sigma^{2}\left(r_{n}\right)}{\sigma^{2}\left(r_{n} / 2\right)} \leq c_{87},
$$

and if $r_{n+1} \geq r_{n} / 2$ we get by virtue of $(9.12)$

$$
\begin{aligned}
\frac{\sigma^{2}\left(r_{n}\right)}{\sigma^{2}\left(r_{n}-r_{n+1}\right)} & \leq c_{88} \exp \left\{2 \int_{r_{n-r_{n+1}}}^{r_{n}} a(s) / s d s\right\} \\
& \leq c_{88} \exp \left\{\frac{2}{\log n} \log \frac{2 r_{n+1}}{r_{n}-r_{n+1}}\right\} \\
& \leq c_{89} .
\end{aligned}
$$


Hence combining (9.10), (9.13) and (9.14) it follows by choosing $c_{86}$ suitably that

$$
\sum_{n}^{\infty} P\left(F^{n}\right)=\sum_{n}^{\infty} \Phi\left(c_{90} \sqrt{\log n}\right)=+\infty,
$$

while by concavity of $\sigma^{2}(x)$ we have

$$
P\left(F^{n} \cap F^{m}\right) \leq P\left(F^{n}\right) P\left(F^{m}\right) .
$$

These imply that

$$
\varlimsup_{n \rightarrow \infty} \frac{X\left(s_{0}+r_{n} e\right)-X\left(s_{0}+r_{n+1} e\right)}{\sigma\left(r_{n}\right) \phi\left(r_{n}\right)} \geq c_{86}
$$

with probability one. By the definition of $\varphi(x)$ and $r_{n}$ we see

$$
\lim _{n \rightarrow \infty} \frac{\varphi\left(r_{n}\right)}{\phi\left(r_{n+1}\right)}=\lim _{n \rightarrow \infty} \sqrt{\frac{\log (n+1)}{\log n}}=1 .
$$

Therefore it follows from (9.17), (9.18) and symmetricity of $X(s)$ that

$$
\varlimsup_{n \rightarrow \infty} \frac{X\left(s_{0}+r_{n} e\right)-X\left(s_{0}\right)}{\sigma\left(r_{n}\right) \varphi\left(r_{n}\right)} \geq c_{\mathrm{s}} / 2
$$

or

$$
\varlimsup_{n \rightarrow \infty} \frac{X\left(s_{0}+r_{n+1} e\right)-X\left(s_{0}\right)}{\sigma\left(r_{n}\right) \phi\left(r_{n}\right)} \geq c_{s 6} / 2
$$

with probability one. Thus we have

$$
\overline{\lim }_{\left\|s-s_{0}\right\| \downarrow 0} \frac{X(s)-X\left(s_{0}\right)}{\sigma\left(\left\|s-s_{0}\right\|\right) \phi\left(\left\|s-s_{0}\right\|\right)} \geq c_{86} / 2 .
$$

Analogously, by choosing $r_{n}=2^{-n}$, we have

$$
\varlimsup_{\left\|s=s_{0}\right\| \downarrow 0} \frac{X(s)-X\left(s_{0}\right)}{\sigma\left(\left\|s-s_{0}\right\|\right) \sqrt{\log _{(2)} 1 /\left\|s-s_{0}\right\|}} \geq c_{91}
$$

with probability one. This completes the proof of Theorem 6 , (ii).

\section{Proofs of Theorems 7 and 8}

Now we shall prove Theorems 7 and 8 by means of following the proofs of Theorems 3 and 4 step by step. An alogous 
consideration in case of Theorem 3, implies that it is sufficient to prove Theorem 7 for $\varphi(x)$ such that

$$
\sqrt{\log _{(2)} 1 / x} \ll \varphi(x) \ll \sqrt{3 \log _{(2)}} 1 / x .
$$

First we assume that $I_{l}(\sigma, \varphi)<+\infty$. One sets

$$
\begin{aligned}
& \varepsilon_{n}=\sigma^{-1}\left(\frac{\sigma\left(2^{-n}\right)}{\sqrt{\log (n \log 2)}}\right) \\
& T_{n}=\left\{s \in D ; 2^{-n-2} \leq\left\|s-s_{0}\right\| \leq 2^{-n-1}\right\} .
\end{aligned}
$$

Let $\left\{t_{i}^{(m)}, i=1, \cdots, N_{\varepsilon_{n}}\left(T_{n}\right)\right\}$ be a minimal $\varepsilon_{n}$-net of $T_{n}$, and set

$$
\begin{aligned}
& S_{n, i}=\left\{s \in T_{n} ;\left\|t_{i}^{(n)}-s\right\| \leq \varepsilon_{n}\right\}, \\
& E_{i}^{n}=\left\{\omega ; \sup _{s \in S_{n, i}} Y(s) \geq \varphi\left(2^{-n-1}\right)+d_{8} F_{\sigma}\left(2 \varepsilon_{n}\right) / \sigma\left(2^{-n-2}-\varepsilon_{n}\right)\right\}, \\
& Y(s)=\frac{X(s)-X\left(s_{0}\right)}{\sigma\left(\left\|s-s_{0}\right\|\right)}, \quad s \in S_{n, i} .
\end{aligned}
$$

Then we have

$$
\begin{aligned}
E\left[(Y(s)-Y(t))^{2}\right] & \leq \frac{\sigma^{2}(\| s-t||)}{\sigma\left(\| s-s_{0}||\right) \sigma\left(\left\|t-s_{0}\right\|\right)} \\
& \leq \sigma^{2}(\| s-t||) / \sigma^{2}\left(2^{-n-2}-\varepsilon_{n}\right)
\end{aligned}
$$

and

$$
\frac{\varphi\left(2^{-n-1}\right)}{\sigma\left(2^{-n-2}-\varepsilon_{n}\right)} \leq d_{24} .
$$

Therefore using Lemma 9 we get

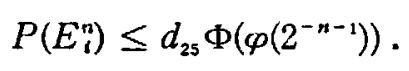

Since

$$
N_{\vartheta_{n}}\left(T_{n}\right) \leq c_{91}\left(2^{-n-1} / \varepsilon_{n}\right)^{N},
$$

we have

$$
\begin{aligned}
\sum_{n}^{\infty} \sum_{i}^{N_{g_{n}\left(T_{n}\right)}} P\left(E_{i}^{n}\right) \leq & \sum_{n}^{\infty} c_{92}\left(2^{-n}-2^{-n-1}\right) \varepsilon_{n}^{-N} 2^{-(n+1)(N-1)} \\
& \times \Phi\left(\varphi\left(2^{-n-1}\right)\right) \\
\leq & c_{93} I,(\sigma, \varphi)<+\infty .
\end{aligned}
$$

This yields $\phi \in \mathcal{U}^{\prime}(X)$ by the same reason as the proof of Theorem 3. 
Next we assume that $I_{l}(\sigma, \varphi)=+\infty$, and one sets

$$
\begin{gathered}
\varepsilon_{n}=\sigma^{-1}\left(\frac{\sigma\left(2^{-n}\right)}{\sqrt{\log (n \log 2)}}\right) \\
S_{n}=\left\{s \in D ; 2^{-n-1} \leq\left\|s-s_{0}\right\| \leq 2^{-n+2}\right\} .
\end{gathered}
$$

Let $\left\{s_{n}^{(n)} ; i=1,2, \cdots, M_{\varepsilon_{n}}\left(S_{n}\right)\right\}$ be a maximal $\varepsilon_{n}$-distinguishable set of $S_{n}$ and set

$$
\begin{gathered}
\left\|s_{i}^{(n)}-s_{0}\right\|=r_{i}^{(n)}, \\
F_{i}^{n}=\left\{\omega ; X\left(s_{i}^{(n)}\right)-X\left(s_{0}\right) \geq \sigma\left(r_{i}^{(n)}\right) \phi\left(r_{i}^{(n)}\right)\right\} .
\end{gathered}
$$

Then we have

$$
P\left(F_{\imath}^{n}\right) \geq \Phi\left(\varphi\left(2^{-n-1}\right)\right),
$$

and

$$
M_{\varepsilon_{n}}\left(S_{n}\right) \leq c_{9_{4}}\left(2^{-n} / \varepsilon_{n}\right)^{N} .
$$

Combining (10.9) and (10.10) it follows that

$$
\begin{aligned}
\sum_{n}^{\infty} \sum_{i=1}^{n_{g n}\left(s_{n}\right)} P\left(F_{i}^{n}\right) & \geq \sum_{n}^{\infty} c_{95} \frac{\left(2^{-n-1}-2^{-n}\right) 2^{-(n-1) \times N-1)} \exp \left\{-\frac{1}{2} \phi^{2}\left(2^{-n+1}\right)\right\}}{\varepsilon_{n}^{N} \varphi\left(2^{-n+1}\right)} \\
& \geq c_{96} I_{t}(\sigma, \varphi)=+\infty .
\end{aligned}
$$

Now we shall check the condition (b) of Lemma 3 (ii). Set

$$
\begin{aligned}
& \gamma_{i}^{(m)}=E\left[\left(X\left(s_{i}^{(n)}\right)-X\left(s_{0}\right)\right)\left(X\left(s_{j}^{(n)}\right)-X\left(s_{0}\right)\right)\right] / \sigma\left(r_{i}^{(n)}\right) \sigma\left(r_{j}^{(m)}\right), \\
& S_{m, k}^{(1)}=\left\{s_{j}^{(m)} ; 1-k / n \leq \gamma_{j}^{(m)} \leq 1-(k-1) / n\right\}, \quad 1 \leq k \leq \sqrt{\log n}+1, \\
& S_{m}^{(2)}=\left\{s_{j}^{(m)} ; 1 / \varphi\left(r_{i}^{(n)}\right) \varphi\left(r_{j}^{(m)}\right) \leq \gamma_{j}^{(m)} \leq 1-1 / \sqrt{\log n}\right\} \\
& m_{0}=n+3 \log _{(2)} n /(\alpha \log 2),
\end{aligned}
$$

for fixed $n, i$ such that $r_{i}^{(n)} \geq r_{j}^{(m)}$. Since by the concavity of $\sigma^{2}(x)$ we see

$$
\begin{aligned}
\gamma_{j}^{(m)} & =\frac{\sigma^{2}\left(r_{i}^{(m)}\right)+\sigma^{2}\left(r_{j}^{(m)}\right)-\sigma^{2}\left(r_{i}^{(m)}-r_{j}^{(m)}\right)}{2 \sigma\left(r_{i}^{(m)}\right) \sigma\left(r_{j}^{(m)}\right)} \\
& \leq \sigma\left(r_{j}^{(m)}\right) / \sigma\left(r_{i}^{(n)}\right),
\end{aligned}
$$

it follows from (10.1) that

(10.12) $\quad \gamma_{j}^{(m)} \varphi\left(r^{(m)}\right) \varphi\left(r_{j}^{(m)}\right) \leq \sigma\left(2^{-m ! 2}\right) \varphi\left(2^{-n ! 2}\right) \varphi\left(2^{-m+1}\right) / \sigma\left(2^{-n ! 1}\right)$

$\ll 1$. 
Using Lemma 4, (ii) we have

$$
P\left(F_{i}^{n} \cap F_{j}^{m}\right) \leq d_{3} P\left(F_{i}^{n}\right) P\left(F_{j}^{m}\right)
$$

for $m \geq m_{0}$. While there exists a constant $c_{97}$ such that

$$
\begin{aligned}
\gamma_{j}^{(m)} & \leq \sigma\left(r_{j}^{(m)}\right) / \sigma\left(r_{i}^{(n)}\right) \\
& \leq \sigma\left(2^{-n+c_{97}+2}\right) / \sigma\left(2^{-n \cdot 1}\right) \ll 1 / 2
\end{aligned}
$$

and from concavity of $\sigma^{2}(x)$ we have

(10.15) $\gamma_{j}^{(m)}$

$$
\begin{aligned}
& \leq 1-\frac{\sigma^{2}\left(r_{i}^{(n)}-r_{j}^{(n)}\right)-\sigma^{2}\left(r_{j}^{(m)}\right)\left(\sqrt{1+\sigma^{2}\left(r_{i}^{(n)}-r_{!}^{(m)}\right) / \sigma^{2}\left(r_{j}^{(m)}\right)}-1\right)^{2}}{2 \sigma\left(r_{i}^{(n)}\right) \sigma\left(r_{j}^{(m)}\right)} \\
& \leq 1-c_{98} \sigma^{2}\left(r_{i}^{(m)}-r_{j}^{(m)}\right) / \sigma^{2}\left(2^{-n+2}\right) \quad \text { for } n \leq m \leq n+c_{9 i} .
\end{aligned}
$$

This yields

$$
\begin{aligned}
\neq S_{m, k}^{(1)} & \leq c_{90}\left(\sigma^{-1}\left(\sqrt{\frac{k}{c_{98}}} \sigma\left(2^{-n+2}\right)\right) / \varepsilon_{m}\right)^{N} \\
& \leq c_{100} k^{\alpha N} \text { for } n \leq m \leq n+c_{97} .
\end{aligned}
$$

Combining (10.14), (10.15) and (10.16) we have

$$
\begin{aligned}
& \left(\sum_{m=n}^{n+c_{97}} \sum_{k=1}^{\sqrt{\log n}+1} \sum_{j \in S_{m, 1}}+\sum_{m=n}^{m_{11}} \sum_{j \in S_{m}^{(2)}}\right) P\left(F_{i}^{n} \cap F_{j}^{m}\right) \\
& \leq\left[c_{9 ;} \sum_{k=32}^{\infty} c_{100} k^{\alpha N} \exp \left\{-c_{101} k\right\}+\frac{3 \log (2) n}{\alpha \log 2}(\log n)^{N / \infty}\right. \\
& \left.\times \exp \left\{-c_{102} \sqrt{\log n}\right\}\right] P\left(F_{i}^{n}\right) \\
& \leq d_{26} P\left(F_{i}^{n}\right) .
\end{aligned}
$$

This yields the proof of Theorem 7 .

The proofs of Corollaries 5, 6 and Theorem 8 are given by the analogous method for Corollaries 3,4 and Theorem 5 . So we omit them.

\section{Proofs of Propositions $1 \sim 4$}

It is obvious from Lemma 5 that if $\sigma(x)$ is a n.r.v.f. there exists a constant $c_{103}$ such that $\sigma(x)<c_{103}<+\infty$ and that if $\sigma(x)$ is a n.s.v.f. we get

$$
\lim _{x \downarrow 0} F_{\sigma}(x) / \sigma(x) \geq \int_{0}^{\infty} \frac{\lim _{x \downarrow 0}}{\frac{\sigma\left(x e^{-u^{2}}\right)}{\sigma(x)}} d u=+\infty
$$


by Fatou's Lemma. This shows Proposition 1.

To prove Propositions 2 and 3 the following Lemma is useful.

Lemma 11. Assume that $\sigma(x)$ is a n.s.v.f. with a structure function a(x) satisfying the conditions (ii) (b) of Theorem 5 and (ii) of Proposition 2. Then

$$
\sigma(x)=F_{\sigma}(x) / \sigma(x) \leq c_{104} / \sqrt{a(x)} .
$$

Proof. By the expression (2.11) of a s.v.f. we get

$$
\begin{aligned}
& \tilde{\sigma}(x) \leq c_{105} \int_{0}^{\infty} \exp \left\{-\int_{x e^{--x^{2}}}^{x} a(s) / s d s\right\} d u \\
= & c_{1 D_{B} \vee} \sqrt{\log 1 / x} \int_{n}^{\infty} \exp \left\{-(\log 1 / x) \int_{1}^{1+y^{2}} a\left(e^{-s \log 1 / s}\right) d s\right\} d y .
\end{aligned}
$$

Set

$$
f_{x}(y)=\int_{1}^{1+y^{2}} a\left(e^{-s \log 1 / x}\right) d s .
$$

For a fixed $x, f_{x}(y)$ is a strictly increasing function of $y$ with continuous positive derivative and so the inverse function $f_{x}^{-1}(z)$ has the same properties. In (11.3) changing the variable $y$ to $z=(\log 1 / x) f_{x}(y),(11.3)$ yields

$$
\begin{aligned}
& \sqrt{\log 1 / x} \int_{0}^{\infty} \exp \left\{-\log (1 / x) f_{x}(y)\right\} d y \\
= & \sqrt{\log 1 / x} \int_{0}^{\infty} e^{-z} d f^{-1}(z / \log 1 / x) .
\end{aligned}
$$

Since by the assumption (ii) (b) of Theorem 5 we get

(11.6) $z=(\log 1 / x) f_{x}(y) \gg \int_{1}^{1+y^{2}} \gamma / s d s=\gamma \log \left(1+y^{2}\right) \quad(x \downarrow 0)$,

it holds that

$$
f_{z}^{-1}(z / \log 1 / x) \ll \exp (z / 2 \gamma), \quad(x \downarrow 0) .
$$

Therefore we have

$$
\int_{0}^{\infty} e^{-z} f_{z}^{-1}(z / \log 1 / x) d z<+\infty .
$$

Integrating by part the right hand of (11.5), we get 


$$
\begin{aligned}
& \sqrt{\log 1 / x} \int_{0}^{\infty} e^{-z} d f_{x}^{-1}(z / \log 1 / x) \\
= & \sqrt{\log 1 / x} \int_{0}^{\infty} e^{-z} f_{x}^{-1}(z / \log 1 / x) d z \\
= & \sqrt{\log 1 / x} \int_{0}^{(\log 1 / x) f_{x}^{(1)}} e^{-z} f_{x}^{-1}(z / \log 1 / x) d z \\
& +\sqrt{\log 1 / x} \int_{(\log 1 / x) f_{x}(1)}^{e^{-z} f_{x}^{-1}(z / \log 1 / x) d z} \\
= & I_{1}+I_{2} .
\end{aligned}
$$

To estimate $I_{1}$, set $f(z /(\log 1 / x))=y$. If $z$ changes a value 0 to $(\log 1 / x) f_{x}(1)$ then $y$ takes a value 0 to 1 . Then by the assumption (ii) of Proposition 2 we have

$$
\frac{z}{\log 1 / x}=f_{x}(y) \geq c_{10_{6}} a(x) y^{2} \quad \text { for } \quad 0 \leq y \leq 1
$$

Hence we have

$$
\begin{aligned}
I_{1} & \ll \sqrt{\log 1 / x} \int_{0}^{(\log 1 / x) f_{x}(1)} e^{-z} \frac{\sqrt{z}}{\sqrt{c_{105}} a(x) \log 1 / x} d z \\
& \leq \frac{\sqrt{\pi}}{2 \sqrt{c_{106} a(x)}} .
\end{aligned}
$$

Next by (11.7) and (11.10) we get

$$
\begin{aligned}
I_{2} & \ll \sqrt{\log 1 / x} \int_{(\log 1 / x)_{c_{106} a(x)}^{\infty}}^{e^{-z-z / z \gamma} d z} \\
& \leq \sqrt{\frac{2 e}{c_{106}}\left(\frac{2 \gamma}{2 \gamma-1}\right)^{3 / 2} \frac{1}{\sqrt{a(x)}} .}
\end{aligned}
$$

Thus combining (11.9), (11.11) and (11.12) one gets the proof of Lemma 11. Proposition 2 is an immediate consequence of Lemma 11. If $\sigma(x)$ satisfies the condition of Proposition 3 we have

$$
\bar{\sigma}(x) 、 \overline{\log 1 / \bar{x}} \leq c_{10 \tau} .
$$

To estimate the lower bound of (11.13), it is sufficient to show the inequality

$$
\begin{aligned}
\bar{\sigma}(x) & \geq G_{\sigma}(\sqrt{\log 1 / x}) / \sigma(x) \\
& \gg c_{108} \sqrt{\log 1 / x},
\end{aligned}
$$

under the assumption of Proposition 3. 
In fact we see

(11. 15)

$$
\begin{aligned}
& G_{\sigma}(\sqrt{\log 1 / x}) / \sigma(x) \\
\geq & c_{109} \int_{\sqrt{\log 1 / x}}^{\infty} \exp \left\{-\frac{c_{4}\left(y^{2}-\log 1 / x\right)}{\log 1 / x}\right\} d y \\
= & \frac{c_{109} e^{c_{4}}}{\sqrt{c_{4}}} \int_{c_{4}}^{\infty} e^{-y^{2}} d y \sqrt{\log 1 / x} .
\end{aligned}
$$

This completes the proof of Proposition 3.

To prove Proposition 4 it is sufficient to show the following Lemma.

Lem ma 12. Assume that $\sigma(x)$ is a n.s.v.f. with a structure function $a(x)$ which satisfies the condition of Proposition 4. Then it follows that

(11. 16) $\quad \sigma(x) \leq c_{110} \vee \overline{\log } 1 / \bar{x}\left(\log _{(2)} 1 / x\right) \cdots\left(\log _{(m)} 1 / x\right)$,

(11. 17) $G_{\pi}(\sqrt{\log 1 / x}) / \sigma(x) \geq c_{111} \sqrt{\log 1 / x}\left(\log _{(2)} 1 / x\right) \cdots\left(\log _{(m)} 1 / x\right)$.

Proof. Set

$$
\begin{gathered}
g(y)=\sqrt{y}(\log y) \cdots\left(\log _{(m-2)} y\right)\left(\log _{(m-1)} y\right)^{c_{7}} \\
\exp (x)=e(x), \quad e^{m}(x)=\exp \left(e^{m-1}(x)\right) .
\end{gathered}
$$

It follows from (11.3) that

$$
\begin{aligned}
\bar{\sigma}(x) & \leq c_{105} \sqrt{\log 1 / x} \int_{0}^{\infty} \exp \left\{-(\log 1 / x) \int_{1}^{1+y^{2}} a\left(e^{-s \log 1 / x}\right) d s\right\} d y \\
& =c_{105} \sqrt{\log 1 / x} \int_{0}^{1} \exp \left\{-\int_{\log 1 / x}^{\left(y^{2}+1\right) \log 1 / x} a\left(e^{-u}\right) d u\right\} d y \\
& +c_{105} \sqrt{\log 1 / x} \int_{1}^{\infty} \exp \left\{-\int_{\log 1 / x}^{\left(y^{2}+1\right) \log 1 / x} a\left(e^{-u}\right) d u\right\} d y \\
& =J_{1}+J_{2} .
\end{aligned}
$$

Obviously we see

$$
J_{1} \leq c_{105} \sqrt{\log 1 / x}
$$

By the condition of Proposition 4, we have

$$
\text { (11.20) } \begin{aligned}
J_{2} & \ll c_{105} \sqrt{\log 1 / \bar{x}} \int_{1}^{\infty} \exp \left\{-\int_{\log 1 / x}^{y 2 \log 1 / x} g^{\prime}(u) / g(u) d u\right\} d y \\
& \leq c_{112} \sqrt{\log 1 / x}\left(\log _{(z)} 1 / x\right) \cdots\left(\log _{(m)} 1 / x\right) .
\end{aligned}
$$


This yields (11. 16).

Finally we shall prove (11.17). It follows that

$$
\begin{aligned}
& G_{\sigma}(\sqrt{\log 1 / x}) / \sigma(x) \\
& \geq c_{113} \int_{\sqrt{\log 1 / x}}^{\infty} \frac{g(\log 1 / x)}{g\left(y^{2}\right)} \exp \left\{-\int_{\log 1 / x}^{y ?}\left(a\left(e^{-u}\right)-\frac{g^{\prime}(u)}{g(u)}\right) d u\right\} d y
\end{aligned}
$$

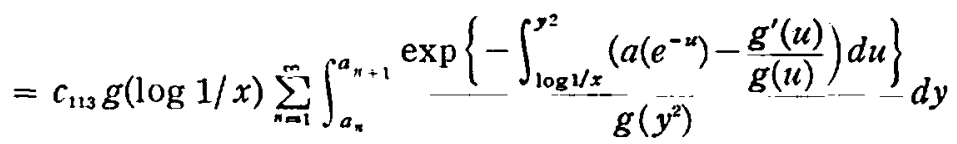

$$
\begin{aligned}
& \gg c_{113} g(\log 1 / x)\left(\log _{(m)} 1 / x\right)_{6}^{c_{6}-c_{7}} \sum_{n=1}^{\infty} \int_{a_{n}}^{a_{n+1}} \frac{\left(\log _{(m-1)} y^{2}\right)^{c_{7}-c_{\theta}}}{g(y)^{2}} d y \\
& \geq c_{114} \sqrt{\log 1 / x}\left(\log _{(2)} 1 / x\right) \cdots\left(\log _{(m)} 1 / x\right) \text {, }
\end{aligned}
$$

where $a_{n}=e\left(\frac{1}{2} e^{m-3}\left(\left(\log _{(m-1)} 1 / x\right)^{n}\right)\right)$.

This completes the proof of Lemma 12 .

\section{Osaka UNIVERSity}

\section{REFERENCES}

[1] Yu. K. Belyaev : Continuity and Hölder conditions for sample functions of stationary Gaussian processes, Proc. of 4 th Berkeley Symp. on Math. Stat. and Prob. II (1961) 23-33.

[2] S. M. Berman : Limit theorems for the maximum term in stationary sequences, Ann. Math. Stat. 35 (1964), 502-516.

[3] K. L. Chung-P. Erdös - T. Sirao: On the Lipschitz's condition for Brow. nian motion, J. Math. Soc. Japan Vol. 11 No. 4 (1959), 263-274.

[4] R.M. Dudley: The sizes of compact subsets of Hilbert space and continuity of Gaussian processes, J. Functional analysis 1 (1967), 290-330.

[5] X. Fernique: Continuité des processis Gaussiens, C. R. Acad. Sci. Paris, t. 258 (1964), 6058-6060.

[6] K. Ito-M. Nisio: On the Convergence of sums of independent Banach space valued variables, Osaka J. Math. 5 (1968), 313-326.

[7] J. Karamata: Sur un mode de croissance reguliere, Theoremes fondamentux, Bulletin de la Soc. Math. de France 61 (1933) 55-62.

[8] T. Kawada: On the upper and lower class for Gaussian processes with several parameters, Nagoya Math. J. Vol. 35 (1969), 109-132.

[9] A.N. Kolmogorov and V.M. Tihomirov : $\varepsilon$-entropy and $\varepsilon$-capacity of sets in functional spaces, Uspehi Mat. Nauk 14 (1954), 3-86.

[10] P. Lévy: Theorie de l'addition des variables aleatoires, Paris, Gauthier. Villars (1937).

[1I] G. G. Lorentz: Metric entropy and approximation, Bull. Amer. Math. Soc. 72 (1966), 903-937,

[12] H. P. McKean, Jr: Stochastic integral, Academic press New York, (1969). 
[13] M. B. Marcus: Hölder conditions for Gaussian processes with stationary increments, Trans. Amer. Math. Soc. Vol. 134 (1968), 29-52.

[14] I. Petrovsky: Zur crsten Randwertaufgabe der Wärmeleitungleichung, Compositio Math. 1 (1935) 383-419.

[15] I. J. Shoenberg: Metric spaces and completely monotonic functions, Annals of Math. Vol. 39 (1938), 811-841.

[16] T. Sirao: On the uniform continuity of Wiener process, J. Math. Soc. Japan $6(1954), 332-335$.

[17] - On the continuity of Brownian motion with a multidimentional parameter, Nagoya Math. J. Vol. 16 (1960), 135-156.

[18] I. Sirao-H. Watanabe: On the Hölder continuity of stationary Gaussian processes, Proc. Japan Academy Vol. 14 (1968), 182-484.

[19] P.T. Strait: Sample function regularity for Gaussian processes with the parameter in a Hilbert space, Pacific J. Math. Vol. 19 (1966), 159-193.

[30] M. Tsuzi: Several topies in analysis (Kaiseki Zatsuron in Japanese), Kyoritsusya Tokyo, 1995.

[21] J. B. Walsh: A note on uniform convergence of stochastic processes, Proc. Amer. Math. Soc. Vol. 18, No. 1 (1967) 129-132. 